hep-th/9708051

\title{
Gluon Condensation in Nonperturbative Flow Equations
}

\author{
M. Reuter \\ DESY, Notkestraße 85, D-22603 Hamburg \\ C. Wetterich \\ Institut für Theoretische Physik \\ Universität Heidelberg \\ Philosophenweg 16, D-69120 Heidelberg
}

\begin{abstract}
:
We employ nonperturbative flow equations for an investigation of the effective action in Yang-Mills theories. We compute the effective action $\Gamma[B]$ for constant color magnetic fields $B$ and examine Savvidy's conjecture of an unstable perturbative vacuum. Our results indicate that the absolute minimum of $\Gamma[B]$ occurs for $B=0$. Gluon condensation is described by a nonvanishing expectation value of the regularized composite operator $F_{\mu \nu} F^{\mu \nu}$ which agrees with phenomenological estimates.
\end{abstract}




\section{Introduction}

A perturbative calculation of the effective action for constant color-magnetic fields $B$ in Yang-Mills theories indicates that the configuration of lowest Euclidean action does not correspond to vanishing fields [1], [2]. This has led to many interesting speculations about the nature of the QCD vacuum. Unfortunately, perturbation theory is clearly invalid in the interesting region in field space. It breaks down both for vanishing magnetic fields and for fields $B$ corresponding to the minimum of the perturbatively calculated effective action $\Gamma[B]$. A nonperturbative computation of $\Gamma[B]$ was lacking so far. In this paper we make an attempt to evaluate $\Gamma[B]$ based on the non-perturbative method of the average action [3]. Within our approximations we find that the minimum of $\Gamma[B]$ occurs for a vanishing magnetic field $B=0$, in contrast to the perturbative result [1], [2]. This strongly suggests that the minimum of the effective action occurs for vanishing gauge fields $A_{\mu}=0$. This implies a vanishing expectation value $<A_{\mu}>=0$ and Lorentz invariance of the $\mathrm{QCD}$ vacuum is trivially realized!.

The vanishing expectation value $\left\langle A_{\mu}>\right.$ does by no means indicate that the QCD vacuum is simple. Gluon condensation phenomena may be described by nonvanishing expectation values of composite operators such as $<F_{\mu \nu} F^{\mu \nu}>$. We also discuss this issue in the context of the average action and compute the expectation value of a suitably regularized operator. Our result for the gluon condensate agrees with phenomenological estimates. (The anomalous trace of the energy momentum tensor induced by the condensate $\left\langle F_{\mu \nu} F^{\mu \nu}>\right.$ is found to be $\left.-0.015 \mathrm{GeV}^{4}\right)$. This may be considered as a first analytical computation of this condensate from "first principles". However, in view of the large possible truncation errors and the un-

\footnotetext{
${ }^{1}$ See ref. 12 for a discussion of possible realizations of Lorentz symmetry for $\left\langle A_{\mu}\right\rangle \neq 0$.
} 
certainty in the precise correspondence between the computed operator and more phenomenological concepts of a gluon condensate it seems premature to draw any quantitative conclusions. We rather consider this work as an encouraging first step towards a quantitatively more reliable computation of the gluon condensate.

The average action $\Gamma_{k}$ is the effective Euclidean action for averages of fields which obtains by integrating out all quantum fluctuations with (generalized) momenta $q^{2}>k^{2}$. It can be viewed as the standard effective action $\Gamma$ computed with an additional infrared cutoff $\sim k$ for all fluctuations. In the limit $k \rightarrow 0$ the average action equals the usual effective action, $\Gamma_{0}=\Gamma$. For $k>0$ no infrared divergences should appear in the computations and a lowering of $k$ allows to explore the longdistance physics step by step. The $k$-dependence of the average action is described by an exact nonperturbative evolution equation [4] The structure of this equation is close to a perturbative one-loop equation but it involves the full propagator and vertices instead of the classical ones. For gauge theories it can be formulated in a way such that $\Gamma_{k}[A]$ is a gauge-invariant functional of the gauge field $A$ [7], [8], [9]. [1]

The flow equation for the pure non-abelian Yang-Mills theory has already been solved [7] with a very simple approximation - the average action $\Gamma_{k}$ has been truncated with a minimal kinetic term $\sim Z_{k} F_{\mu \nu} F^{\mu \nu}$. From the $k$-dependence of $Z_{k}$ the running of the renormalized gauge coupling $g(k)$ has been derived for arbitrary dimension $d$. Most strikingly, this lowest-order estimate suggests that the wave function renormalization $Z_{k}$ reaches zero for $k=k_{\infty}$ and turns negative for $k<k_{\infty}$. Here the scale $k_{\infty}$ can be identified with the confinement scale, i.e., the scale where the renormalized gauge coupling $g^{2}(k) \sim Z_{k}^{-1}$ diverges. This was interpreted as an indication for the instability of the perturbative vacuum with $A_{\mu}=0$, similar to

\footnotetext{
${ }^{2}$ For the relation to earlier versions of exact renormalization group equations [5] see ref. [6].

${ }^{3}$ See ref. [10, 11] for alternative formulations.
} 
the perturbative result [1], [2]. Indeed, negative $Z_{k}$ would imply that the minimum of $\Gamma_{k}$ must occur for nonvanishing gauge field for all $k<k_{\infty}$ [12]. It is obvious, however, that a truncated effective action $\sim Z_{k} B^{2}$ is insufficient to describe such phenomena. One expects positive $\Gamma_{k}$ for large $B$ and this cannot be accommodated with a negative $Z_{k}$ in the "lowest order truncation".

In this paper we enlarge the "space of actions" by considering for $\Gamma_{k}$ an arbitrary function of constant magnetic fields, i.e., $\Gamma_{k} \sim W_{k}\left(\frac{1}{2} B^{2}\right)$. The flow equation describes how the function $W_{k}$ changes its shape, starting from a linear dependence $W_{k}=\frac{1}{2} Z_{k} B^{2}$ for large $k$. The approximations (truncations) employed for the computation and solution of the flow equation are summarized at the end of sect. 2 . In particular, we solve numerically the flow equations in a truncation where $W_{k}$ is approximated by a polynomial of order $B^{6}$. We find that in contrast to the perturbative computation of ref. [1] the minimum of $W_{k}$ remains at $B=0$ for all $k$. The vanishing of $Z_{k}$ for $k_{\infty}>0$ turns out to be an artifact of the lowest order truncation. In the truncation of order $B^{6}$ the gauge coupling grows large but remains finite. In this approximation the ground state value of the gauge field $A_{\mu}$ vanishes, in contrast to speculations in ref. [12].

For the description of gluon condensation in terms of the expectation value of a suitably regularized gauge-invariant composite operator $F_{\mu \nu} F^{\mu \nu}$ we use exact flow equations including composite operators [13]. Following this approach we enlarge in sect. 4 our truncation by studying a general function $V_{k}\left(\frac{1}{2} B^{2}, \chi\right)$, where $\chi$ is a composite field related to the operator $F_{\mu \nu} F^{\mu \nu}$. We find that $\chi$ develops indeed an expectation value which is stable for $k \rightarrow 0$. This implies a nonvanishing gluon condensate.

In the following we shall consider the flow equation for the pure $S U(N)$ YangMills theory. The general formalism of our formulation with ghost fields and back- 
ground fields $\bar{A}$ is briefly reviewed in appendix A, to which the reader could turn at this point. There we write down the corresponding modified Slavnov-Taylor identities [11] and a further identity which describes the dependence of the effective action on the background field $\bar{A}_{\mu}$ [14], [15]. We also give in this appendix the full exact flow equation including the ghost sector. Since we are interested in the ground state and there exist always classical solutions with vanishing ghost fields, we concentrate here on the zero ghost sector. Then the effective action reduces to a functional $\Gamma_{k}[A, \bar{A}]$ depending only on the gauge field $A$ and the background field $\bar{A}$. It is invariant under simultaneous gauge transformations of both fields. With a suitable truncation for the ghost dependence of the full effective action (cf. appendix A) one obtains the following renormalization group equation for the scale dependence of $\Gamma_{k}[A, \bar{A}](t \equiv \ln k)$ :

$$
\begin{aligned}
\frac{\partial}{\partial t} \Gamma_{k}[A, \bar{A}]= & \frac{1}{2} \operatorname{Tr}_{x c L}\left[\left(\Gamma_{k}^{(2)}[A, \bar{A}]+R_{k}\left(\Gamma_{k}^{(2)}[\bar{A}, \bar{A}]\right)\right)^{-1} \frac{\partial}{\partial t} R_{k}\left(\Gamma_{k}^{(2)}[\bar{A}, \bar{A}]\right)\right] \\
& -\operatorname{Tr}_{x c}\left[\left(-D^{\mu}[A] D_{\mu}[\bar{A}]+R_{k}\left(-D^{2}[\bar{A}]\right)\right)^{-1} \frac{\partial}{\partial t} R_{k}\left(-D^{2}[\bar{A}]\right)\right]
\end{aligned}
$$

Here the first trace on the r.h.s. arises from the fluctuations of the gauge field. It involves on integration over space-time (" $x$ ") as well as a summation over color ("c") and Lorentz (" $L$ ") indices. The "color" trace is in the adjoint representation. The second trace is due to the Faddeev-Popov ghosts and is over space-time and color indices only. The precise form of the infrared cutoff is described by the function $R_{k}$. It is convenient to choose a smooth cutoff which vanishes for large covariant momenta

$$
R_{k}(u)=u\left[\exp \left(\mathcal{Z}_{k}^{-1} u / k^{2}\right)-1\right]^{-1}
$$

For the purpose of comparison we will also consider in this paper a second choice for the cutoff, namely simply a constant

$$
R_{k}=\mathcal{Z}_{k} k^{2}
$$


In eqs. (1.2), (1.3) the wave function renormalization $\mathcal{Z}_{k}$ could be a matrix in the space of fields which may even depend on $\bar{A}$. (In ref. [7] we used $\mathcal{Z}_{k} \equiv 1$ for the ghosts and a $k$-dependent constant $\mathcal{Z}_{k} \equiv Z_{F, k}$ for all modes of the gauge field.) Eq. (1.1) is a functional differential equation where $\Gamma_{k}^{(2)}[A, \bar{A}]$ denotes the matrix of second functional derivatives of $\Gamma_{k}$ with respect to $A$, with the background field $\bar{A}$ kept fixed. The modes of the gauge field are declared "high-frequency modes" or "low-frequency modes" depending on whether their eigenvalues with respect to the operator $\left.\Gamma_{k}^{(2)}[\bar{A}, \bar{A}] \equiv \Gamma_{k}^{(2)}[A, \bar{A}]\right|_{A=\bar{A}}$ are larger or smaller than $k^{2}$, respectively. Therefore it is this operator which appears in the argument of $R_{k}$ t and in this sense $R_{k}$ acts as an effective infrared cutoff by suppressing the low frequency modes.

In this paper we aim for an approximate solution of the flow equation (1.1) by a truncation of the most general form of $\Gamma_{k}[A, \bar{A}]$. We derive in sect. 2 the evolution equation for the effective action for constant color magnetic fields $B$. This equation is further approximated in sect. 3 by using a polynomial ansatz for $\Gamma_{k}(B)$ of order $B^{6}$ and solving it numerically. In sect. 4 we enlarge the truncation by introducing a field for the composite operator $F_{\mu \nu} F^{\mu \nu}$. This will permit the investigation of the flow of the expectation value of $F_{\mu \nu} F^{\mu \nu}$ and an extraction of the gluon condensate for $k \rightarrow 0$. Sect. 5 finally contains our conclusions and a brief discussion of further possible developments. Since some of the necessary computations are technically

\footnotetext{
${ }^{4}$ In [7] we used the classical $S^{(2)}[\bar{A}, \bar{A}]$ rather than $\Gamma_{k}^{(2)}[\bar{A}, \bar{A}]$ for this purpose. While preserving all the general properties of $\Gamma_{k}$, the new flow equation is much easier to handle from a technical point of view. This change also entails the different positioning of $\mathcal{Z}_{k}$ in (1.2) relative to the one in [7]: for the simple truncation used there one has $\Gamma_{k}^{(2)}=Z_{F, k} S^{(2)}$ for part of the modes. We only will use the definition (1.2) if for high momenta $q^{2} \rightarrow \infty$ one has $u \rightarrow \infty$. We should mention, however, that for negative eigenvalues of $\Gamma_{k}^{(2)}[\bar{A}, \bar{A}]$ the vanishing of $R_{k}$ for $k \rightarrow 0$ is guaranteed only if the ratio $u / k^{2}$ in (1.2) remains finite. This problem concerns mainly the approach to convexity of the effective action for $k \rightarrow 0$ and is of no relevance for the present work.
} 
involved, we display a lot of this material in various appendices.

\section{Evolution equation of the effective action for constant color-magnetic fields}

In order to find nonperturbative approximative solutions of (1.1) we employ the following ansatz for $\Gamma_{k}$ :

$$
\Gamma_{k}[A, \bar{A}]=\int d^{d} x W_{k}\left(\frac{1}{4} F_{\mu \nu}^{z}(x) F_{z}^{\mu \nu}(x)\right)+\frac{1}{2 \alpha_{k}} \int d^{d} x \sum_{z}\left(D_{\mu}[\bar{A}]\left(A^{\mu}-\bar{A}^{\mu}\right)\right)_{z}^{2}
$$

Here $W_{k}$ is an arbitrary function of the invariant $\frac{1}{4} F^{2}$ with $F_{\mu \nu}^{z}$ the field strength of the gauge field $A$. The $k$-dependence of $W_{k}$ will be determined by inserting (2.1) into the evolution equation (1.1). If we think of $W_{k}(\theta)$,

$$
\theta \equiv \frac{1}{4} F_{\mu \nu}^{z} F_{z}^{\mu \nu}
$$

as a power series in $\theta$ our ansatz contains at this point still invariants of arbitrarily high canonical dimension. Since all invariants which occur are of the form $\theta^{l}$, only the dimensions $4 l, l=1,2, \ldots$, actually occur in this truncation. Also, for a fixed dimension $4 l$ the truncation (2.1) does not contain a complete basis of operators. Nevertheless one may hope that (2.1) gives a qualitatively correct picture of the effective action for constant color magnetic fields in the regime where the renormalization group evolution has already drastically modified the classical Lagrangian $\frac{1}{4} F^{2}$. We remark that effective actions which depend on $\theta$ only play also a central role in the leading-log models [17] of QCD.

The second term on the r.h.s. of (2.1) is a standard background gauge-fixing term [16], [7] with a $k$-dependent gauge-fixing parameter $\alpha_{k}$. In addition to the $k$-dependence of the function $W_{k}(\theta)$ we should, in principle, also compute the $k$ dependence of $\alpha_{k}$. We will omit this here since the general identities of appendix A 
imply that $\alpha$ is independent of $k$ in a first approximation. Furthermore, we will see that within the truncation (2.1) and for a suitable choice of the infrared cutoff $R_{k}$ the evolution equation for $W_{k}$ becomes independent of $\alpha_{k}$.

We should mention at this place that a truncation is actually not completely defined by the terms retained but rather by specifying which invariants in the most general form of $\Gamma_{k}$ are omitted. One may parametrize a general $\Gamma_{k}$ by infinitely many couplings multiplying the infinitely many possible invariants which can be formed from the gauge fields consistent with the symmetries. In the corresponding infinite dimensional space a truncation is a projection on a subspace which is defined by setting all but the specified couplings to zero. (In our case the subspace remains infinite dimensional.) In practice, we will choose a particular test configuration $A_{\mu}$ corresponding to a constant magnetic field. The truncation should then be understood in the sense that we use a basis for the invariants where all invariants except those used in (2.1) vanish for the test configuration. By putting the coefficients of all invariants which vanish for the test configuration to zero the truncation is uniquely defined. A computation of $W_{k}$ therefore amounts to a computation of the $k$-dependent effective action for a (particular) constant magnetic field.

As a particularly convenient test field we choose a covariantly constant colormagnetic field [2] with a vector potential of the form

$$
A_{\mu}^{z}(x)=n^{z} \mathrm{~A}_{\mu}(x)
$$

Here $n^{z}$ is a constant unit vector in color space $\left(n^{z} n_{z}=1\right)$, and $\mathrm{A}_{\mu}(x)$ is any "abelian" gauge field whose field strength

$$
\mathrm{F}_{\mu \nu}=\partial_{\mu} \mathrm{A}_{\nu}-\partial_{\nu} \mathrm{A}_{\mu}=B \epsilon_{\mu \nu}^{\perp}=\text { const }
$$

corresponds to a constant magnetic field $B$ along the 3 -direction, say. (We define $\epsilon_{12}^{\perp}=-\epsilon_{21}^{\perp}=1$, with all other components vanishing.) Hence we have $\theta=$ 
$\frac{1}{4} F_{\mu \nu}^{z} F_{z}^{\mu \nu}=\frac{1}{2} B^{2}$

In summary, the solution of the evolution equation will produce the effective action for constant color magnetic fields of the type (2.3, 2.4), where an additional infrared cutoff $k$ is present. One is finally interested in the limit $k \rightarrow 0$. For the computation of this effective action we make approximations which amount to the following truncations of the effective action:

1. The ghost sector is approximated by its classical form, as discussed in appendix A.

2. The remaining gauge field dependence of $\Gamma_{k}$ is approximated by (2.1).

3. The resulting flow equation which will be derived in this section is a nonlinear partial differential equation for a function of two variables, $W_{k}(\theta) \equiv W(k, \theta)$. We solve numerically in sect. 3 only an approximate version of this differential equation where $W_{k}(\theta)$ is truncated to a polynomial in $\theta$ of degree three.

We do not expect these approximations to yield a quantitatively precise result in the range of $k$ where the gauge coupling is large. Nevertheless, it remains an interesting question if the speculated effect of an unstable perturbative vacuum persists in this picture. Our findings indicate, in contrast to perturbation theory [1], [2] , that the minimum of the effective action occurs for $A_{\mu}=0$. We emphasize that despite the approximations made our approach goes far beyond the perturbative calculation of refs. [1], [2]. We conclude that there is no reason to believe that the configuration $B=0$ (or $A_{\mu}=0$ ) is unstable.

The evolution equation for $W_{k}(\theta)$ is computed for arbitrary dimension $d$ in appendix B. One finds

$$
\frac{\partial}{\partial t} W_{k}\left(\frac{1}{2} B^{2}\right)=\frac{1}{2} \Omega^{-1} \operatorname{Tr}_{x c L}\left[H\left(W_{k}^{\prime} \mathcal{D}_{T}\right)\right]
$$




$$
\begin{aligned}
& +\frac{1}{2} \Omega^{-1} \operatorname{Tr}_{x c}\left[\tilde{H}\left(-\alpha_{k}^{-1} D^{2}\right)-H\left(-W_{k}^{\prime} D^{2}\right)\right]-\Omega^{-1} \operatorname{Tr}_{x c}\left[H_{G}\left(-D^{2}\right)\right] \\
& +v_{d}\left(\frac{1}{W_{k}^{\prime}+B^{2} W_{k}^{\prime \prime}}-\frac{1}{W_{k}^{\prime}}\right)\left(\frac{1}{W_{k}^{\prime}}\right)^{\frac{d}{2}-1} \int_{0}^{\infty} d x x^{\frac{d}{2}-1} H(x) \\
& -v_{d} \int_{0}^{\infty} d x x^{\frac{d}{2}-1}\left(\tilde{H}\left(\frac{x}{\alpha_{k}}\right)-H\left(\frac{x}{\alpha_{k}}\right)\right)
\end{aligned}
$$

with $W_{k}^{\prime} \equiv\left(\partial W_{k} / \partial \theta\right)\left(\frac{1}{2} B^{2}\right)$, etc., $\Omega=\int d^{d} x$, and

$$
v_{d}^{-1} \equiv 2^{d+1} \pi^{\frac{d}{2}} \Gamma\left(\frac{d}{2}\right)
$$

Here we have introduced the convenient abbreviation (for $\mathcal{Z}_{k}=Z_{k}$ )

$$
\begin{aligned}
H(u) \equiv & \left(u+R_{k}(u)\right)^{-1} \frac{\partial}{\partial t} R_{k}(u) \\
& = \begin{cases}\left(2+\frac{d}{d t} \ln Z_{k}\right) \frac{u}{Z_{k} k^{2}}\left[\exp \left(\frac{u}{Z_{k} k^{2}}\right)-1\right]^{-1} & \text { for } \\
\left(2+\frac{d}{d t} \ln Z_{k}\right) Z_{k} k^{2}\left[u+Z_{k} k^{2}\right]^{-1} & \text { for }\end{cases}
\end{aligned}
$$

In the second, ghost-type, trace the function $H_{G}(u)$ is defined similarly, but with a different factor $\mathcal{Z}_{k}=1$. The last trace accounts for a possible difference between $\tilde{Z}_{k}$ and $Z_{k}$ in $(B .20)$. Here $\tilde{H}$ is obtained from $H$ by replacing $Z_{k}$ by $\tilde{Z}_{k}$.

The eigenvalues of the operator

$$
\mathcal{D}_{T} \equiv-D^{2}+2 i \bar{g} F
$$

are known explicitly [2]. They are parametrized by a $(d-2)$-dimensional momentum $q^{\mu}$ which "lives" in the space orthogonal to the 1-2 plane, and a discrete quantum number $n=0,1,2, \ldots$ which labels the Landau levels. The spectral sum for the function $\hat{H}(x) \equiv H\left(W_{k}^{\prime} x\right)$ reads $^{5}$

$$
\Omega^{-1} \operatorname{Tr}_{x c L}\left[\hat{H}\left(\mathcal{D}_{T}\right)\right]=\sum_{l=1}^{N^{2}-1} \frac{\bar{g}\left|\nu_{l}\right| B}{2 \pi} \sum_{n=0}^{\infty} \int \frac{d^{d-2} q}{(2 \pi)^{d-2}}
$$

\footnotetext{
${ }^{5}$ The momentum integration is absent for $d=2$.
} 


$$
\begin{aligned}
& \cdot\left\{(d-2) \hat{H}\left(q^{2}+(2 n+1) \bar{g}\left|\nu_{l}\right| B\right)\right. \\
& +\hat{H}\left(q^{2}+(2 n+3) \bar{g}\left|\nu_{l}\right| B\right) \\
& \left.+\hat{H}\left(q^{2}+(2 n-1) \bar{g}\left|\nu_{l}\right| B\right)\right\}
\end{aligned}
$$

Here $\nu_{l}, l=1, \ldots, N^{2}-1$ are the eigenvalues of the matrix $n^{z} T_{z}$ in the adjoint representation. We note that for $n=0$ and $q^{2}$ sufficiently small the eigenvalue $q^{2}-\bar{g}\left|\nu_{l}\right| B$ in the third term on the r.h.s. of $(2.8)$ can become negative. This instability [1], 18] causes severe problems if one tries to compute the standard oneloop effective action in the background of a covariantly constant magnetic field 19. In our approach this problem is cured by the presence of an IR regulator. Eq. (2.8) can be rewritten $(d>2)$ as

$$
\begin{aligned}
& \Omega^{-1} \operatorname{Tr}_{x c L}\left[\hat{H}\left(\mathcal{D}_{T}\right)\right]=\frac{v_{d-2}}{\pi} \sum_{l=1}^{N^{2}-1} \bar{g}\left|\nu_{l}\right| B \int_{0}^{\infty} d x x^{\frac{d}{2}-2} \\
& \cdot\left\{d \sum_{n=0}^{\infty} \hat{H}\left(x+(2 n+1) \bar{g}\left|\nu_{l}\right| B\right)+\hat{H}\left(x-\bar{g}\left|\nu_{l}\right| B\right)-\hat{H}\left(x+\bar{g}\left|\nu_{l}\right| B\right)\right\}
\end{aligned}
$$

but it cannot be simplified any further in closed form. The other traces in (2.5) are given by

$$
\Omega^{-1} \operatorname{Tr}_{x c}\left[\hat{H}\left(-D^{2}\right)\right]=\frac{v_{d-2}}{\pi} \sum_{l=1}^{N^{2}-1} \bar{g}\left|\nu_{l}\right| B \int_{0}^{\infty} d x x^{\frac{d}{2}-2} \sum_{n=0}^{\infty} \hat{H}\left(x+(2 n+1) \bar{g}\left|\nu_{l}\right| B\right)
$$

and similarly for $\tilde{H}$ and $H_{G}$.

In this paper we use two different methods in order to (approximately) compute the spectral sums (2.9) and (2.10). In appendix $\mathrm{F}$ we shall represent them as Schwinger proper-time integrals [20]. This method leads to compact integral representations which are valid for all values of $B$, but it has the disadvantage that it works only for the cutoff function $R_{k}(x)$ defined by (A.31) which leads, as we shall see, to ultraviolet problems.

The second method consists of expanding the r.h.s. of (2.9) in powers of $B$. It is applicable if $\bar{g} B \ll k^{2}$. It works for any function $R_{k}(x)$ such that ultraviolet 
problems can easily be avoided. The condition $\bar{g} B \ll k^{2}$ guarantees that we may express the sum over $n$ by an Euler-McLaurin series, and that the terms become small rapidly. We will concentrate mainly on the second approach. In this manner (2.9) turns into

$$
\begin{aligned}
& \Omega^{-1} \operatorname{Tr}_{x c L}\left[H\left(W_{k}^{\prime}\left(\frac{1}{2} B^{2}\right) \mathcal{D}_{T}\right)\right] \\
& =\left(N^{2}-1\right) \frac{d v_{d-2}}{2 \pi}\left[W_{k}^{\prime}\left(\frac{1}{2} B^{2}\right)\right]^{-\frac{d}{2}} \int_{0}^{\infty} d x \int_{0}^{\infty} d y x^{\frac{d}{2}-2} H(x+y) \\
& +\frac{v_{d-2}}{\pi} \sum_{m=1}^{\infty} C_{m}^{d}\left(\sum_{l=1}^{N^{2}-1} \nu_{l}^{2 m}\right)(\bar{g} B)^{2 m}\left[W_{k}^{\prime}\left(\frac{1}{2} B^{2}\right)\right]^{2 m-\frac{d}{2}} \int_{0}^{\infty} d x x^{\frac{d}{2}-2} H^{(2 m-1)}(x)
\end{aligned}
$$

with

$$
C_{m}^{d}=\frac{d}{(2 m) !}\left(2^{2 m-1}-1\right) B_{2 m}-\frac{2}{(2 m-1) !}
$$

Here $B_{2 m}$ are the Bernoulli numbers. In a second step one has to expand the $B^{2}$-dependence of $W_{k}^{\prime}$. Note that only even powers of $B$ occur in this expansion. The group-theoretical factors $\sum_{l=1}^{N^{2}-1} \nu_{l}^{2 m}$ are discussed in appendices $\mathrm{C}$ and $\mathrm{D}$. In particular for $S U(2)$ these factors equal 2 for all values of $m$.

If we use the Euler-McLaurin series (2.11) and a similar expansion for the trace (2.10) in eq. (2.5), we find for $S U(N)$ :

$$
\begin{aligned}
& \frac{\partial}{\partial t} W_{k}(\theta)=\frac{v_{d-2}}{2 \pi}(2-\eta) k^{d}\left\{\frac{d-1}{2}\left(N^{2}-1\right) r_{2}^{d}\left(\frac{W_{k}^{\prime}}{Z_{k}}\right)^{-\frac{d}{2}}\right. \\
& \left.-\sum_{m=1}^{\infty} \tau_{m}\left(C_{m}^{d}-E_{m}\right) r_{0}^{d, m}\left(\frac{2 \bar{g}^{2} \theta}{k^{4}}\right)^{m}\left(\frac{W_{k}^{\prime}}{Z_{k}}\right)^{2 m-\frac{d}{2}}\right\} \\
& +\frac{v_{d-2}}{2 \pi}(2-\tilde{\eta})\left(\tilde{Z}_{k} \alpha_{k}\right)^{\frac{d}{2}} k^{d}\left\{\frac{1}{2}\left(N^{2}-1\right) r_{2}^{d}-\sum_{m=1}^{\infty} \tau_{m} E_{m} r_{0}^{d, m}\left(\frac{2 \bar{g}^{2} \theta}{k^{4}}\right)^{m}\left(\tilde{Z}_{k} \alpha_{k}\right)^{-2 m}\right\} \\
& -\frac{v_{d-2}}{\pi} k^{d}\left\{\left(N^{2}-1\right) r_{2}^{d}-2 \sum_{m=1}^{\infty} \tau_{m} E_{m} r_{0}^{d, m}\left(\frac{2 \bar{g}^{2} \theta}{k^{4}}\right)^{m}\right\} \\
& +v_{d}(2-\eta) r_{1}^{d} k^{d}\left(\frac{Z_{k}}{W_{k}^{\prime}+2 \theta W_{k}^{\prime \prime}}-\frac{Z_{k}}{W_{k}^{\prime}}\right)\left(\frac{W_{k}^{\prime}}{Z_{k}}\right)^{1-\frac{d}{2}}+\text { const. }
\end{aligned}
$$

with $\tau_{m}$ defined in appendix $\mathrm{D}$ and the constants $E_{m}$ given by

$$
E_{m}=\frac{1}{(2 m) !}\left(2^{2 m-1}-1\right) B_{2 m}
$$


The dimensionless integrals

$$
\begin{aligned}
r_{0}^{d, m} & =-\frac{1}{2-\eta}\left(Z_{k} k^{2}\right)^{2 m-\frac{d}{2}} \int_{0}^{\infty} d x x^{\frac{d}{2}-2} H^{(2 m-1)}(x) \\
& =-\int_{0}^{\infty} d x x^{\frac{d}{2}-2}\left(\frac{d}{d x}\right)^{2 m-1} \frac{x}{e^{x}-1} \\
r_{1}^{d} & =\frac{1}{2-\eta}\left(Z_{k} k^{2}\right)^{-\frac{d}{2}} \int_{0}^{\infty} d x x^{\frac{d}{2}-1} H(x)=\int_{0}^{\infty} d x \frac{x^{\frac{d}{2}}}{e^{x}-1} \\
r_{2}^{d} & =\frac{1}{2-\eta}\left(Z_{k} k^{2}\right)^{-\frac{d}{2}} \int_{0}^{\infty} d x \int_{0}^{\infty} d y x^{\frac{d}{2}-2} H(x+y) \\
& =\int_{0}^{\infty} d x \int_{0}^{\infty} d y \frac{x^{\frac{d}{2}-2}(x+y)}{\exp (x+y)-1}
\end{aligned}
$$

occur as a consequence of (2.7). The second equality in eqs. (2.15) uses the exponential cutoff (1.2). For this choice we note for later use that in 4 dimensions

$$
r_{0}^{4, m}=B_{2 m-2}, r_{1}^{4}=r_{2}^{4}=2 \zeta(3)
$$

where $\zeta$ denotes the Riemann zeta function. The evolution equation (2.13) is the central result of this section. It constitutes a partial differential equation for a function of two variables, $W(\theta, k)$.

In eq. (2.13) we have introduced the anomalous dimensions

$$
\eta=-\frac{d}{d t} \ln Z_{k}, \quad \tilde{\eta}=-\frac{d}{d t} \ln \tilde{Z}_{k}
$$

A convenient choice for the wave function renormalization constants used in the

\footnotetext{
${ }^{6}$ In contrast, the quantities $r_{1}^{4}$ and $r_{2}^{4}$ are not well defined for the choice (A.31). The ultraviolet divergence indicates an incomplete "thinning out" of the high momentum degrees of freedom for a simple mass like infrared cutoff. Even though eq. (2.13) was derived by choosing a specific background field, this evolution equation does not depend on the background we used for the calculation. By employing derivative expansion techniques [21], [22] it should also be possible to derive (2.13) without ever specifying a background. In the case at hand the method presented here is by far simpler, however.
} 
infrared cutoff $R_{k}$ is

$$
Z_{k}=\left\{\begin{array}{l}
W_{k}^{\prime}(0) \quad \text { for } \quad k>k_{n p} \\
W_{k_{n p}}^{\prime}(0) \quad \text { for } \quad k<k_{n p} \\
\tilde{Z}_{k}=\frac{1}{\alpha_{k}}
\end{array}\right.
$$

where $k_{n p}$ is a typical momentum scale which characterizes the transition from the perturbative to the nonperturbative regime. We account for the possibility that $W_{k}^{\prime}(0)$ may turn negative for $k$ smaller than a "confinement scale" $k_{\infty}$, whereas $Z_{k}$ must always be strictly positive. More precisely, if $\left|d \ln W_{k}^{\prime}(0) / d t\right|$ becomes of order unity for small scales $k$, we choose $k_{n p}$ to be the scale where $\left|\eta\left(k_{n p}\right)\right|=1.5$. For $k<k_{n p}$ all couplings run fast anyhow, and an improvement of the scaling properties of $R_{k}$ by the introduction of a $k$-dependent wave function renormalization seems not necessary. The choice $\tilde{Z}_{k}=\alpha_{k}^{-1}$ guarantees that the infrared cutoff acts on the longitudinal modes in the same way as on the transversal modes. It implies that the flow equation for $\frac{\partial}{\partial t} W_{k}(\theta)$ becomes independent ${ }^{7}$ of $\alpha_{k}$ and therefore independent of the "gauge fixing" in our truncation! In this paper we can therefore neglect the running of $\alpha_{k}$, and one has $\tilde{\eta}=0$.

It is convenient to express the flow equation in terms of renormalized dimensionless quantities

$$
\begin{aligned}
g^{2} & =k^{d-4} Z_{k}^{-1} \bar{g}^{2} \\
\vartheta & =g^{2} k^{-d} Z_{k} \theta
\end{aligned}
$$

${ }^{7} \mathrm{~A} \theta$-independent constant in $W_{k}$ is irrelevant.

${ }^{8}$ This can be inferred from a first-order approximation to the solution of the general identities which govern the dependence of $\Gamma_{k}[A, \bar{A}]$ on the background field $\bar{A}[14,[15]$. 


$$
w_{k}(\vartheta)=g^{2} k^{-d} W_{k}(\theta)
$$

Switching to a notation where dots denote derivatives with respect to $\vartheta$ instead of $\theta$ and with $\partial / \partial t$ now taken at fixed $\vartheta$ one obtains

$$
\begin{aligned}
& \frac{\partial}{\partial t} w_{k}(\vartheta)=-(4-\eta) w_{k}(\vartheta)+4 \vartheta \dot{w}_{k}(\vartheta) \\
& +(2-\eta) v_{d} g^{2}\left(\dot{w}_{k}(\vartheta)\right)^{-\frac{d}{2}}\left\{\frac{(d-1)(d-2)}{2}\left(N^{2}-1\right) r_{2}^{d}\right. \\
& \left.-\frac{2 r_{1}^{d} \vartheta \ddot{w}_{k}(\vartheta)}{\dot{w}_{k}(\vartheta)+2 \vartheta \ddot{w}_{k}(\vartheta)}-(d-2) \sum_{m=1}^{\infty} \tau_{m}\left(C_{m}^{d}-E_{m}\right) r_{0}^{d, m}\left(2 \vartheta \dot{w}_{k}^{2}(\vartheta)\right)^{m}\right\} \\
& +2(d-2) v_{d} g^{2} \sum_{m=1}^{\infty} \tau_{m} E_{m} r_{0}^{d, m}(2 \vartheta)^{m}+\mathrm{const}
\end{aligned}
$$

This nonlinear partial differential equation for the function $w(\vartheta, t)$ does not show an explicit $t$ - (or $k$ )-dependence of the right-hand side any more.

One needs in addition the running of the renormalized gauge coupling $g$ and the anomalous dimension $\eta$, which are related by

$$
\beta_{g^{2}}=\frac{\partial g^{2}}{\partial t}=(d-4+\eta) g^{2}
$$

For $k>k_{n p}$ we have by definition $\dot{w}_{k}(0)=1$ and $\eta$ can be determined by

$$
\begin{aligned}
& \frac{\partial}{\partial t} \dot{w}_{k}(0)=0=\eta-2(d-2) v_{d} r_{0}^{d, 1} \tau_{1} g^{2}\left((2-\eta) C_{1}^{d}-(4-\eta) E_{1}\right) \\
& -(2-\eta) v_{d} g^{2}\left(2 r_{1}^{d}+\frac{d(d-1)(d-2)}{4}\left(N^{2}-1\right) r_{2}^{d}\right) \ddot{w}_{k}(0)
\end{aligned}
$$

With $\tau_{1}=N, C_{1}^{d}=\frac{d}{12}-2, E_{1}=\frac{1}{12}$ this yields

$$
\begin{aligned}
\eta=- & \left(\frac{N}{3} v_{d}(d-2)(26-d) r_{0}^{d, 1} g^{2}-2 h_{d} g^{2} w_{2}\right) \\
& \left(1-\frac{N}{6} v_{d}(d-2)(25-d) r_{0}^{d, 1} g^{2}+h_{d} g^{2} w_{2}\right)^{-1}
\end{aligned}
$$

where

$$
h_{d}=v_{d}\left(2 r_{1}^{d}+\frac{d(d-1)(d-2)}{4}\left(N^{2}-1\right) r_{2}^{d}\right)
$$




$$
w_{2}=\ddot{w}_{k}(0)
$$

For general $d$ the constants $r_{0}^{d, 1}, r_{1}^{d}$ and $r_{2}^{d}$ depend on the precise choice of the infrared cutoff except for $d=4$ where $r_{0}^{4,1}=1$ is cutoff independent. The running of the renormalized gauge coupling $g$ is now fully determined by eq. (2.21). It depends on the additional coupling $w_{2}(2.25)$ which will be discussed in more detail in the next section?.

Specifying the initial value $g^{2}(\Lambda)$ and the function $w_{\Lambda}(\vartheta)$ at some high momentum scale $\Lambda$ the form of $w_{k}(\vartheta)$ and $g^{2}(k)$ are completely determined by the flow equation (2.20). Solving for $k \rightarrow 0$ the function $w_{0}(\vartheta)$ specifies the effective action in our truncation. If necessary, one has to replace for $k<k_{n p}$ eq. (2.23) by $\eta=0, g^{2}(k<$ $\left.k_{n p}\right)=g^{2}\left(k_{n p}\right)$.

Before closing this section, we briefly comment on the range of convergence of the Euler-McLaurin series in our case. For large $m$ one has

$$
\lim _{m \rightarrow \infty} C_{m}^{d}=d \lim _{m \rightarrow \infty} E_{m} \sim \pi^{-2 m}
$$

For $N=2$ and $d=4$ we find (cf. (2.16) ) that the coefficients of $(2 \vartheta)^{m}$ in eq. (2.20) diverge $\sim \pi^{-2 m} B_{2 m-2} \sim \pi^{-4 m} 2^{-2 m}(2 m-4)$ !. For small nonvanishing $\vartheta$ the first terms of the series have a very rapid apparent convergence, but the series finally diverges due to the factorial growth $\sim(2 m-4)$ !. We can therefore safely use this series only for the derivatives $w^{(n)}(\vartheta=0)$ with finite $n$ where convergence problems are absent since only a finite number of terms in the sum contributes. The situation is probably similar for $N>2$ and/or $d \neq 4$ as well as for many other choices of the infrared cutoff. In contrast, the original sums over $n$ in eqs. (2.9), (2.10) always converge since for $B>0$ the contributions from sufficiently high values of $n$ are

\footnotetext{
${ }^{9}$ For $w_{2}=0$ we recover the result of ref. [7] except for the factor $(25-d)$ in the denominator in (2.23) which was $(24-d)$ previously. This difference is due to a slightly different choice of the $Z$-factors in the infrared cutoff.
} 
exponentially suppressed. An explicit evaluation of these sums is possible for the simplified masslike IR-cutoff (A.31). This is described in appendix F where we will also see the reason for the ultraviolet divergence of $r_{1}^{4}$ and $r_{2}^{4}$ for this particular cutoff.

\section{Polynomial truncations}

In this section we concentrate on $d=4$ with the exponential infrared cutoff (1.2). One could solve the flow equation (2.20) numerically. Instead, we further simplify here the truncation in order to get a first idea of the physical contents of (2.13). We include in $w_{k}(\vartheta)$ only terms which are at most quadratic in $\vartheta$ :

$$
\begin{gathered}
w_{k}(\vartheta)=w_{0}(k)+w_{1}(k) \vartheta+\frac{1}{2} w_{2}(k) \vartheta^{2}+\frac{1}{6} w_{3}(k) \vartheta^{3} \\
w_{j}(k) \equiv\left(\frac{d}{d \vartheta}\right)^{j} w_{k \mid \vartheta=0}
\end{gathered}
$$

Thus the truncation for $\Gamma_{k}$ is parametrized (up to an irrelevant constant) by three couplings:

$$
\begin{aligned}
\Gamma_{k}[A, A] & =\int d^{d} x\left\{\frac{1}{4} \frac{\bar{g}^{2} w_{1}(k)}{g^{2}(k)} F_{\mu \nu}^{z} F_{z}^{\mu \nu}+\frac{1}{32} \frac{\bar{g}^{4} w_{2}(k)}{g^{2}(k) k^{4}}\left(F_{\mu \nu}^{z} F_{z}^{\mu \nu}\right)^{2}\right. \\
& \left.+\frac{1}{384} \frac{\bar{g}^{6} w_{3}(k)}{g^{2}(k) k^{8}}\left(F_{\mu \nu}^{z} F_{z}^{\mu \nu}\right)^{3}\right\}
\end{aligned}
$$

The short distance or "classical" theory is specified for $k=\Lambda$ by $\left(Z_{\Lambda}=1\right)$

$$
g^{2}(\Lambda)=\bar{g}^{2}, \quad w_{1}(\Lambda)=1, \quad w_{2}(\Lambda)=w_{3}(\Lambda)=0
$$

For $k>k_{n p}$ it follows from the definitions (2.18) and (2.19) that $w_{1}(k)=1$, and $g^{2}(k)$ is determined by eqs. (2.21), (2.23). For $k<k_{n p}$ we use $g^{2}(k)=g^{2}\left(k_{n p}\right)$ instead and keep

$$
w_{1}(k) \equiv \dot{w}_{k}(0)
$$


as the independent running coupling constant The flow equations for the partial derivatives $w_{j}(k) \equiv w_{k}^{(j)}(\vartheta=0)$ follow by differentiating eq. (2.20) with respect to $\vartheta$. For example, one has

$$
\begin{aligned}
& \frac{\partial}{\partial t} \dot{w}=\eta \dot{w}+4 \vartheta \ddot{w}-(2-\eta) v_{d} g^{2} \dot{w}^{-\frac{d}{2}} \\
& \left\{(d-2) \sum_{m=1}^{\infty} \tau_{m}\left(C_{m}^{d}-E_{m}\right) r_{0}^{d, m}\left(2 \vartheta \dot{w}^{2}\right)^{m-1}\left(2 m \dot{w}^{2}+(4 m-d) \vartheta \dot{w} \ddot{w}\right)\right. \\
& +r_{1}^{d}\left(\frac{2 \ddot{w}+2 \vartheta w^{(3)}}{\dot{w}+2 \vartheta \ddot{w}}-\frac{2 \vartheta \ddot{w}\left(3 \ddot{w}+2 \vartheta w^{(3)}\right)}{(\dot{w}+2 \vartheta \ddot{w})^{2}}-d \frac{\vartheta(\ddot{w})^{2}}{\dot{w}(\dot{w}+2 \vartheta \ddot{w})}\right) \\
& \left.+\frac{1}{4} d(d-1)(d-2)\left(N^{2}-1\right) r_{2}^{d} \frac{\ddot{w}}{\dot{w}}\right\} \\
& +4(d-2) v_{d} g^{2} \sum_{m=1}^{\infty} m \tau_{m} E_{m} r_{0}^{d, m}(2 \vartheta)^{m-1}
\end{aligned}
$$

We observe that $w_{0}$ does not appear on the r.h.s. of the evolution equations for $w_{j}, j \geq 1$, and we omit in the following this irrelevant constant.

For $k>k_{n p}$ the evolution equation for the running gauge coupling reads $(d=4)$

$$
\frac{\partial g^{2}}{\partial t}=-\frac{g^{4}}{24 \pi^{2}}\left(11 N-3 H_{4} w_{2}\right)\left[1-\frac{g^{2}}{32 \pi^{2}}\left(7 N-2 H_{4} w_{2}\right)\right]^{-1}
$$

whereas for $k<k_{n p}$ one uses the flow equation for $w_{1}$

$$
\frac{\partial}{\partial t} w_{1}=\frac{g^{2}\left(k_{n p}\right)}{8 \pi^{2}}\left(\frac{11 N}{3}-H_{4} \frac{w_{2}}{w_{1}^{3}}\right)
$$

where $H_{4}=16 \pi^{2} h_{4}=r_{1}^{4}+3\left(N^{2}-1\right) r_{2}^{4}=2\left(3 N^{2}-2\right) \zeta(3)$ for the choice (1.2). It is obvious that for $w_{2}<0$ the gauge coupling $g^{2}(k)$ always increases (3.6) until at $k=k_{n p}$ the anomalous dimension $|\eta|$ reaches 1.5. If $w_{2}$ remains negative for $k<k_{n p}$, the coupling $w_{1}$ decreases until it reaches zero at the confinement scale $k_{\infty}>0$. (If we define $g^{2}(k)=g^{2}\left(k_{n p}\right) / w_{1}$, this coupling diverges at the confinement scale.) The issue is different for $w_{2}>0$ : The coupling $w_{1}$ does not reach zero since for small enough $w_{1}$ the second term in eq. (3.7) would cancel the first term. One therefore needs an estimate of $w_{2}(k)$. 
Let us first consider the regime $k>k_{n p}$ where the evolution equation for $w_{2}$ reads (with $E_{2}=-\frac{7}{720}, C_{2}^{4}=-\frac{67}{180}$ )

$$
\begin{aligned}
& \frac{\partial}{\partial t} w_{2}=(4+\eta) w_{2}+\frac{g^{2}}{8 \pi^{2}}\left\{\tau_{2} r_{0}^{4,2}\left(\frac{127}{45}-\frac{29}{20} \eta\right)\right. \\
& \left.+(2-\eta)\left(5 r_{1}^{4}+\frac{9}{2}\left(N^{2}-1\right) r_{2}^{4}\right) w_{2}^{2}-(2-\eta)\left(r_{1}^{4}+\frac{3}{2}\left(N^{2}-1\right) r_{2}^{4}\right) w_{3}\right\}
\end{aligned}
$$

We observe the appearance of the coupling $w_{3}$ whose evolution is given by (for details see appendix F)

$$
\begin{aligned}
& \frac{\partial}{\partial t} w_{3}=(8+\eta) w_{3}+\frac{g^{2}}{16 \pi^{2}}\left\{-\frac{1}{30}\left(\frac{442}{315}-\frac{137}{210} \eta\right) \tau_{3}\right. \\
& \left.+\frac{87}{30}(2-\eta) \tau_{2} w_{2}-6 \zeta(3)(2-\eta)\left[\left(12 N^{2}+23\right) w_{2}^{3}-\left(9 N^{2}+8\right) w_{2} w_{3}+N^{2} w_{4}\right]\right\}
\end{aligned}
$$

We neglect the term $\sim w_{4}$ which is consistent with our approximation. In the perturbative region, where $N g^{2} / 16 \pi^{2}$ is small, it is easy to infer from (3.8) that $w_{2}$ is of the order $g^{2}$. In fact, we may neglect $\eta$ and the terms $\sim w_{2}^{2}$ and $\sim w_{3}$ in the curly bracket in (3.8). In lowest order one finds for the ratio $\frac{w_{2}}{g^{2}}$ an infrared stable fixed point:

$$
\begin{gathered}
\frac{\partial}{\partial t}\left(\frac{w_{2}}{g^{2}}\right)=4 \frac{w_{2}}{g^{2}}+\frac{127}{360 \pi^{2}} \tau_{2} r_{0}^{4,2} \\
w_{2 *}(k)=-\frac{127}{1440 \pi^{2}} \tau_{2} r_{0}^{4,2} g^{2}(k)=-\frac{127}{270} \frac{g^{2}}{16 \pi^{2}}
\end{gathered}
$$

which is approached very rapidly. (The last equality in (3.11) holds for $N=2$ and uses $r_{0}^{4,2}=\frac{1}{6}$ for the exponential cutoff.) Similarly, one obtains the lowest order fixed point

$$
w_{3 *}(k)=\frac{221 \tau_{3}}{37800} \frac{g^{2}(k)}{16 \pi^{2}}
$$

Actually, as we show in Appendix F, all ratios $w_{n} / g^{2}$ reach perturbative fixed points for $n \geq 3$. This justifies the approximation (3.1) at least for small enough $g^{2}(k)$. (We observe $W_{n} \sim g^{2(n-1)} k^{-4(n-1)}$.) 
It is interesting to insert the value (3.11) into the $\beta$-function for $g^{2}$ eq. (3.6). Expanding in powers of $g^{2}$ one has

$$
\begin{aligned}
& \frac{\partial g^{2}}{\partial t}=-\frac{22 N}{3} \frac{g^{4}}{16 \pi^{2}}-\frac{77 N^{2}}{3} \frac{g^{6}}{\left(16 \pi^{2}\right)^{2}}+\left(3 N^{2}-2\right) \zeta(3) \frac{g^{4}}{4 \pi^{2}} w_{2} \\
& =-\frac{22}{3} \frac{g^{4} N}{16 \pi^{2}}-\left(\frac{77}{3}+\frac{127}{45} \zeta(3) \tau_{2}\left(1-\frac{2}{3 N^{2}}\right)\right) \frac{g^{6} N^{2}}{\left(16 \pi^{2}\right)^{2}}
\end{aligned}
$$

We note that without the term $\sim w_{2}$ the coefficient $\sim g^{6}$ exceeds the perturbative two-loop coefficient $-\frac{204}{9} \frac{N^{2}}{\left(16 \pi^{2}\right)^{2}}$ only by a little more than $10 \%$. It is recomforting to find the contribution from $w_{2}$ in the same order of magnitude as this difference. We emphasize that a full computation of $\beta_{g^{2}}$ in order $g^{6}$ should take additional invariants into account, as for example $(F \tilde{F})^{2}$ or $\left(D_{\mu} F^{\mu \nu}\right)^{2}$. We also observe that for $k=k_{n p}$, i.e., for $\eta=-\frac{3}{2}$ one has approximately $N g^{2} / 16 \pi^{2}=\frac{1}{7}$ so that the validity of perturbation theory extends roughly to all $k>k_{n p}$.

Let us finally consider the regime $k<k_{n p}$. Our truncation permits, in principle, a first-order type transition where the absolute minimum of $w$ jumps from $\vartheta=0$ to a nonzero value $\vartheta_{0}>0$. For positive $w_{3}$ the polynomial (3.1) is bounded from below for $\vartheta \geq 0$. For $w_{1}>0$ there is always a local minimum at $\vartheta=0$. Two additional extrema are present if

$$
w_{2}^{2}>2 w_{1} w_{3}
$$

There is a minimum for positive $\vartheta_{0}$

$$
\vartheta_{0}=\frac{-w_{2}+\sqrt{w_{2}^{2}-2 w_{1} w_{3}}}{w_{3}}
$$

and, for $w_{1}>0$, a maximum at

$$
\vartheta_{\max }=\frac{-w_{2}-\sqrt{w_{2}^{2}-2 w_{1} w_{3}}}{w_{3}}
$$

For $w_{1}<0$ the origin $\vartheta=0$ turns to a local maximum. The critical set of couplings where the two minima are of equal height $\left(w_{k}\left(\vartheta_{0}\right)=0\right)$ corresponds to

$$
w_{1}=\frac{3}{8} \frac{w_{2}^{2}}{w_{3}}
$$


For $w_{3}$ smaller than the critical value, the absolute minimum occurs at $\vartheta_{0}$. This would correspond to the picture where the perturbative vacuum is unstable, i.e. $<A_{\mu}>\neq 0$.

For $k>k_{n p}$ where $w_{1}=1$ an inspection of the flow equations (cf. (3.11), (3.12)) shows that $2 w_{3}$ remains larger than $w_{2}^{2}$. There is therefore only one minimum at $\vartheta=0$. If $w_{2}$ remains negative for $k<k_{n p}$, the coupling $w_{1}$ would decrease towards zero. If furthermore $w_{3}$ stays positive, the condition (3.17) would then necessarily be met for some scale $k>k_{\infty}$. On the other hand, if $w_{2}$ turns positive, $w_{1}$ will also remain strictly positive, and the only minimum occurs at $\vartheta=0$ for all values of $k$.

We have solved numerically the system of flow equations for $k<k_{n p}$

$$
\begin{aligned}
& \frac{\partial}{\partial t} w_{2}=4 w_{2}+\frac{g^{2}\left(k_{n p}\right)}{16 \pi^{2} w_{1}^{2}}\left\{\tau_{2}\left(\frac{29}{30} w_{1}^{4}-\frac{7}{270} w_{1}^{2}\right)\right. \\
& \left.+4 \zeta(3)\left[\left(9 N^{2}+1\right) \frac{w_{2}^{2}}{w_{1}^{2}}-\left(3 N^{2}-1\right) \frac{w_{3}}{w_{1}}\right]\right\} \\
& \frac{\partial}{\partial t} w_{3}=8 w_{3}+\frac{g^{2}\left(k_{n p}\right)}{16 \pi^{2} w_{1}^{2}}\left\{-\frac{\tau_{3}}{30}\left(\frac{137}{105} w_{1}^{6}+\frac{31}{315} w_{1}^{2}\right)+\frac{87}{15} \tau_{2} w_{1}^{3} w_{2}\right. \\
& \left.-12 \zeta(3)\left(N^{2} \frac{w_{4}}{w_{1}}-\left(9 N^{2}+8\right) \frac{w_{2} w_{3}}{w_{1}^{2}}+\left(12 N^{2}+23\right) \frac{w_{2}^{3}}{w_{1}^{3}}\right)\right\}
\end{aligned}
$$

together with eq. (3.7) for $w_{1}$. (According to our truncation, we put $w_{4}=0$.) For $N=3$ we find that $w_{3}$ remains positive for all $k$, whereas $w_{2}$ changes sign for $k \approx 0.5 k_{n p} \approx \Lambda_{\mathrm{QCD}}$. In consequence, all three couplings run to fixed values as $k \rightarrow 0$, given by

$$
w_{1 *}=0.13, \quad w_{2 *}=4 \cdot 10^{-4}, \quad w_{3 *}=4 \cdot 10^{-6}
$$

At least within the $F^{6}$ truncation we find that the ground state occurs for $A_{\mu}=0$, contradicting earlier speculations and the estimates of too simple $F^{2}$ or $F^{4}$ truncations! As an immediate consequence, the gauge coupling never diverges and

$$
\alpha_{s} \equiv g^{2}\left(k_{n p}\right) /\left(4 \pi w_{1}\right)
$$


reaches the value

$$
\alpha_{s}(k \rightarrow 0)=3.35
$$

\section{Gluon condensate}

Let us now turn to the gluon condensate as described by the expectation value of a suitably smeared composite operator $\sim<F_{\mu \nu} F^{\mu \nu}>$. This expectation value may not vanish despite a vanishing ground state value for the gauge field $\left.<A^{\mu}\right\rangle=0$. The formalism for the introduction of composite fields in the flow equations is described in [13], and we use it here in the version without a separate infrared cutoff for the composite field $\chi$. Essentially, this amounts to the introduction of an identity of the type

$$
1=\mathrm{const} \int \mathcal{D} \chi \exp \left[-\int d^{d} x \frac{1}{2 a k_{\chi}^{4}}\left\{k_{\chi}^{3} \chi-\frac{1}{4} F_{\mu \nu}^{z} F_{z}^{\mu \nu}\right\}^{2}\right]
$$

into the functional integral defining the effective average action $\Gamma_{k \chi}$. The scale $k_{\chi}$ and the parameter $a$ should be chosen conveniently such that a maximum of the effects of higher invariants can be described by the dynamics of the new scalar singlet $\chi$. Typically $k_{\chi}$ should be a scale where the influence of $w_{2}$ and $w_{3}$ on the running of $w_{1}$ (or $g^{2}$ ) becomes important. The expectation value $<\chi>$ for $k \rightarrow 0$ is directly related [13] to the expectation value of the composite operator $F_{\mu \nu} F^{\mu \nu}$

$$
<\chi>=\frac{1}{4} k_{\chi}^{-3}<F_{\mu \nu}^{z} F_{z}^{\mu \nu}>.
$$

The operator appearing on the r.h.s. is regulated by smearing over distances $\sim k_{\chi}^{-1}$. For scales $k<k_{\chi}$ we now have to deal with the coupled system of $A_{\mu}$ and $\chi$. The exact flow equation has the same general structure as before, except for the extension of $\Gamma_{k}^{(2)}$ to the second functional derivative including also the new field $\chi$. Nevertheless, since in the formulation used here $R_{k}$ acts only on gauge fields and

ghosts, only the projection of $\left(\Gamma_{k}^{(2)}+R_{k}\right)^{-1}$ on this restricted space enters the flow 
equations. Formally, the components $R_{\chi \chi}$ and $R_{\chi A}$ vanish in the generalized trace on the r.h.s. of the flow equation.

Including the composite field, we extend our truncation for the average action (2.1) by the addition of a term

$$
\Gamma_{k}[A, \chi]=\int d^{d} x\left\{V_{k}\left(\frac{1}{4} F_{\mu \nu}^{z} F_{z}^{\mu \nu}, \chi\right)+\frac{1}{2} Z_{\chi, k}(\chi) \partial^{\mu} \chi \partial_{\mu} \chi\right\}
$$

At the scale $k_{\chi}$, eq. (4.1) implies the "initial values" 13

$$
\begin{aligned}
V_{k \chi}\left(\frac{1}{4} F_{\mu \nu}^{z} F_{z}^{\mu \nu}, \chi\right) & =W_{k \chi}\left(\frac{1}{4} F_{\mu \nu}^{z} F_{z}^{\mu \nu}\right)+\frac{k_{\chi}^{2}}{2 a} \chi^{2}, \\
& -\frac{1}{4 a k_{\chi}} \chi F_{\mu \nu}^{z} F_{z}^{\mu \nu}+\frac{1}{32 a k_{\chi}^{4}}\left(F_{\mu \nu}^{z} F_{z}^{\mu \nu}\right)^{2} \\
Z_{\chi, k_{\chi}}(\chi) & =0
\end{aligned}
$$

The expectation value $\langle\chi\rangle$ for $\left\langle A_{\mu}\right\rangle=0$ corresponds to the minimum of the effective scalar potential

$$
U_{k}(\chi)=V_{k}(0, \chi)
$$

for $k \rightarrow 0$. At the scale $k_{\chi}$ this potential is simply quadratic, $U_{k_{\chi}}=\frac{1}{2 a} k_{\chi}^{2} \chi^{2}$, and has its minimum for $\chi=0$. We will be interested in the change of shape of $U_{k}$ as $k$ flows towards zero.

As in the preceding sections we will be concerned with configurations of spaceindependent static magnetic fields where $\theta=\frac{1}{4} F_{\mu \nu}^{z} F_{z}^{\mu \nu}$ is a constant. We note that $V_{k_{\chi}}(\theta, \chi=0)$ differs from $W_{k_{\chi}}(\theta)$ by the subtraction of terms $\sim \theta^{2}, \theta^{3}$ and $\theta^{4}$. For all $k$, the original function $W_{k}(\theta)$ can be recovered by solving the field equation for $\chi, \frac{\partial V}{\partial \chi}\left(\theta, \chi_{0}(\theta)\right)=0$, and inserting $\chi_{0}(\theta)$ into $V$, i.e. $W(\theta) \equiv V\left(\theta, \chi_{0}(\theta)\right)$. Nevertheless, the introduction of $\chi$ effectively extends the truncation and therefore results in modified flow equations for $W_{k}(\theta)$. In particular, we can now expand $V_{k}(\theta, \chi)$ in powers of $\theta$ for arbitrary constant $\chi$. 
Let us next derive the evolution equation for the scale dependence of $V_{k}$. This is done by evaluating the general flow equation for constant $\chi$ and $B$, inserting on the r.h.s. the truncation (4.3). We need the inverse propagator $\Gamma_{k}^{(2)}$ for this "background" configuration. The components $\left(\Gamma_{k}^{(2)}\right)_{A_{\mu} A_{\nu}}$ remain the same as in the previous sections, if we simply replace $W_{k}(\theta)$ by $V_{k}(\theta, \chi)$. The pure scalar piece is also easily obtained

$$
\left(\Gamma_{k}^{(2)}\right)_{\chi \chi}=\frac{1}{2} \frac{\partial^{2} V_{k}(\theta, \chi)}{\partial \chi^{2}}+\tilde{Z}_{\chi, k}(\chi)\left(-\partial^{2}\right) .
$$

We note, however, that the scalar fluctuations can influence the flow equation only indirectly through the off diagonal piece $\left(\Gamma_{k}^{(2)}\right)_{\chi A_{\mu}}$. Using

$$
\frac{\delta \Gamma_{k}}{\delta A_{\mu}^{z}(x)}=\int d^{d} z \frac{\delta \theta(z)}{\delta A_{\mu}^{z}(x)} \frac{\partial V_{k}}{\partial \theta}(z)=\left(D_{\nu} F^{\mu \nu}\right)_{z}(x) \frac{\partial V_{k}}{\partial \theta}(x)
$$

we find for the constant magnetic field background

$$
\frac{\delta^{2} \Gamma_{k}}{\delta A_{\mu}^{z}(x) \delta \chi(y)}=\left(D_{\nu} F^{\mu \nu}\right)_{z}(x) \frac{\delta}{\delta \chi(y)} \frac{\partial V_{k}}{\partial \theta}(x)=0 .
$$

For this configuration we therefore find a particularly simple flow equation: Since $\left(\Gamma_{k}^{(2)}+R_{k}\right)$ is block diagonal and $R_{\chi \chi}=0$, it reduces to the same flow equation as before, with $W_{k}(\theta)$ replaced by $V_{k}(\theta, \chi)$ !

Introducing according to (2.19)

$$
v_{k}(\vartheta, \chi)=g^{2} k^{-d} V_{k}(\theta, \chi)
$$

the evolution equation for $v_{k}$ is given by (2.20), with $w_{k}(\vartheta)$ replaced by $v_{k}(\vartheta, \chi)$ and dots denoting partial derivatives with respect to $\vartheta$ at fixed $\chi$. Typically we will choose $k_{\chi}=k_{n p}$ such that $g^{2}(k) \equiv g^{2}\left(k_{n p}\right)$. Expanding as before in cubic order in $\vartheta$

$$
v_{k}(\vartheta, \chi)=u(\chi)+v_{1}(\chi) \vartheta+\frac{1}{2} v_{2}(\chi) \vartheta^{2}+\frac{1}{6} v_{3}(\chi) \vartheta^{3}
$$

${ }^{10} \tilde{Z}_{\chi}(\chi)$ is related to $Z_{\chi}(\chi)$ and its derivative in a simple way. 
the flow equations for $v_{1}(\chi), v_{2}(\chi)$ and $v_{3}(\chi)$ are given by (3.7) and (3.18), with the replacement $w_{i} \rightarrow v_{i}(\chi)$. The evolution of $u(\chi)$ follows from (2.20) for $\vartheta=0$ and $d=4$, i.e.

$$
\frac{\partial}{\partial t} u=-4 u+\frac{3 \zeta(3)}{8 \pi^{2}}\left(N^{2}-1\right) \frac{g^{2}\left(k_{n p}\right)}{v_{1}^{2}} .
$$

The system of equations (4.11), (3.7), and (3.18) still remains a complicated system of four coupled partial differential equations for the functions $u(\chi, t), v_{1}(\chi, t)$, $v_{2}(\chi, t)$ and $v_{3}(\chi, t)$ which depend on two variables.

In order to gain some intuition about this system let us first look at the differential equation for the scalar potential $U_{k}(\chi)$ and its derivatives

$$
\begin{aligned}
\frac{\partial}{\partial t} U & =\frac{3 \zeta(3)}{8 \pi^{2}}\left(N^{2}-1\right) k^{4} v_{1}^{-2} \\
\frac{\partial}{\partial t}\left(\frac{\partial U}{\partial \chi}\right) & =-\frac{3 \zeta(3)}{4 \pi^{2}}\left(N^{2}-1\right) k^{4} v_{1}^{-3} \frac{\partial v_{1}}{\partial \chi} \\
\frac{\partial}{\partial t}\left(\frac{\partial^{2} U}{\partial \chi^{2}}\right) & =\frac{3 \zeta(3)}{4 \pi^{2}}\left(N^{2}-1\right) k^{4} v_{1}^{-4}\left[3\left(\frac{\partial v_{1}}{\partial \chi}\right)^{2}-v_{1} \frac{\partial^{2} v_{1}}{\partial \chi^{2}}\right] .
\end{aligned}
$$

At the scale $k_{\chi}$ the initial values for $v_{1}$ and $\partial v_{1} / \partial \chi$ are given by

$$
\begin{aligned}
v_{1_{\mid k_{\chi}}} & =w_{1 \mid k_{\chi}}-\frac{g^{2}\left(k_{n p}\right)}{\bar{g}^{2} a k_{\chi}} \chi \\
{\frac{\partial v_{1}}{\partial \chi}}_{\mid k_{\chi}} & =-\frac{g^{2}\left(k_{n p}\right)}{\bar{g}^{2} a k_{\chi}}
\end{aligned}
$$

whereas $\partial^{2} v_{1} / \partial \chi_{\mid k_{\chi}}^{2}$ vanishes. The r.h.s. of eq. (4.13) starts therefore positive and induces a negative linear term in $U$ at $\chi=0$, resulting in a minimum at $\chi_{0}(k)>0$ for $k<k_{\chi}$. Simultaneously, the positive r.h.s. of eq. (4.14) leads to a decrease of the mass term for $\chi$. One can follow the $k$-dependence of the minimum value $\chi_{0}(k)\left(\chi_{0} \equiv \chi_{0}(\vartheta=0)\right)$ using the identity

$$
\frac{\partial \chi_{0}}{\partial t}=-m_{\chi}^{-2} \frac{\partial}{\partial t}\left(\frac{\partial U_{k}}{\partial \chi}\right)\left(\chi_{0}\right)
$$

where we have defined the scalar mass term

$$
m_{\chi}^{2} \equiv \frac{\partial^{2} U}{\partial \chi^{2}}\left(\chi_{0}\right)
$$


Inferring the r.h.s. from eqs. (4.13), (4.14) evaluated at $\chi=\chi_{0}$ we conclude that the running of $\chi_{0}$ stops for small $k$ due to the factor $k^{4}$, provided $m_{\chi}^{2}$ and $v_{1}$ remain nonzero for $k \rightarrow 0$.

Let us next consider an expansion in powers of $\vartheta$ for $\chi$ at the minimum $\chi_{0}(k)$ and compare

$$
\begin{aligned}
v_{n} & \equiv v_{n}\left(\chi_{0}\right)=\frac{\partial^{n}}{\partial \vartheta^{n}} v\left(\vartheta, \chi_{0}(\vartheta=0)\right)_{\mid \vartheta=0} \\
w_{n} & \equiv \frac{\partial^{n}}{\partial \vartheta^{n}} w(\vartheta=0)=\frac{d^{n}}{d \vartheta^{n}} v\left(\vartheta, \chi_{0}(\vartheta)\right)_{\mid \vartheta=0}
\end{aligned}
$$

Whereas $w_{1}=v_{1}$ holds for all $k$ the corresponding relation for $w_{2}$ reads

$$
w_{2}=v_{2}-\frac{\partial^{2} u}{\partial \chi^{2}}\left(\chi_{0}\right){\frac{\partial \chi_{0}(\vartheta)}{\partial \vartheta}}_{\mid \vartheta=0} .
$$

For positive $\chi_{0}$ and negative $\frac{\partial v_{1}(\chi)}{\partial \chi}$ we observe $v_{1} \equiv v_{1}\left(\chi_{0}\right)<v_{1}(0)$. Due to the running $\chi_{0}$ the flow equation for $v_{1}$ obtains an additional contribution

$$
\frac{\partial}{\partial t} v_{1}=\frac{g^{2}\left(k_{n p}\right)}{8 \pi^{2}}\left(\frac{11 N}{3}-H_{4} \frac{v_{2}}{v_{1}^{3}}\right)+\frac{\partial v_{1}(\chi)}{\partial \chi}{ }_{\mid \chi_{0}} \frac{\partial}{\partial t} \chi_{0}
$$

and similar for all $v_{n} \equiv v_{n}\left(\chi_{0}\right)$ where a term $\frac{\partial v_{n}(\chi)}{\partial \chi}{ }_{\mid \chi_{0}} \frac{\partial \chi_{0}}{\partial t}$ should be added on the r.h.s. of the flow equations.

It is instructive to compare the flow equation for $v_{1}$ to the one without a condensate (3.7). In the vicinity of $k_{\chi}$ we can use the initial values 4.17), together with

$$
\begin{aligned}
& v_{2_{\mid k_{\chi}}}=w_{2_{\mid k_{\chi}}}+\frac{g^{2}\left(k_{n p}\right)}{a \bar{g}^{4}} \\
& v_{3_{\mid k_{\chi}}}=w_{3_{\mid k_{\chi}}} \\
& m_{\chi \mid k_{\chi}}^{2}=\frac{k_{\chi}^{2}}{a}
\end{aligned}
$$

As compared to eq. (3.7) the difference between $v_{2}$ and $w_{2}$ subtracts from the r.h.s. of (4.20) a piece $g^{4}\left(k_{n p}\right) H_{4} /\left(8 \pi^{2} \bar{g}^{4} a v_{1}^{3}\right)$. On the other hand, the contribution 
$\sim \frac{\partial v_{1}}{\partial \chi} \partial \chi_{0} / \partial t=g^{4}\left(k_{n p}\right)\left(H_{4}-r_{1}^{4}\right) /\left(8 \pi^{2} \bar{g}^{4} a v_{1}^{3}\right)$ almost cancels this piece except for the negative contribution $\sim r_{1}^{4}$. The flow of $w_{1}$ is therefore somewhat slower than the one corresponding to (4.9).

We also need flow equations for the quantities

$$
y_{n}=\frac{\partial v_{n}(\chi)}{\partial \chi}\left(\chi_{0}\right)=\frac{\partial}{\partial \chi} \frac{\partial^{n}}{\partial \vartheta^{n}} v_{\mid \vartheta=0, \chi=\chi_{0}}
$$

which appear on the r.h.s. of the flow equations for $v_{n}, \chi_{0}$ and $m_{\chi}^{2}$. They obtain by partial differentiation of $(4.9)$ and (5.8) with respect to $\chi$ :

$$
\begin{aligned}
& \frac{\partial}{\partial t} y_{1}=\frac{H_{4} g^{2}\left(k_{n p}\right)}{8 \pi^{2}}\left(3 \frac{v_{2} y_{1}}{v_{1}^{4}}-\frac{y_{2}}{v_{1}^{3}}\right) \\
& \frac{\partial}{\partial t} y_{2}=4 y_{2}+\frac{g^{2}\left(k_{n p}\right)}{4 \pi^{2}}\left\{\frac{29}{10} \tau_{2} r_{0}^{4,2} v_{1} y_{1}+\left(5 r_{1}^{4}+\frac{9}{2}\left(N^{2}-1\right) r_{2}^{4}\right)\left(2 \frac{v_{2} y_{2}}{v_{1}^{4}}-4 \frac{v_{2}^{2} y_{1}}{v_{1}^{5}}\right)\right. \\
& \left.-\left(r_{1}^{4}+\frac{3}{2}\left(N^{2}-1\right) r_{2}^{4}\right)\left(\frac{y_{3}}{v_{1}^{3}}-3 \frac{v_{3} y_{1}}{v_{1}^{4}}\right)\right\} \\
& \frac{\partial}{\partial t} y_{3}=8 y_{3}+\frac{g^{2}\left(k_{n p}\right)}{16 \pi^{2}}\left\{\frac{548}{105} \tau_{3} r_{0}^{4,3} v_{1}^{3} y_{1}+\frac{174}{5} \tau_{2} r_{0}^{4,2}\left(v_{1} y_{2}+v_{2} y_{1}\right)\right. \\
& +6 r_{1}^{4}\left(17 \frac{v_{2} y_{3}}{v_{1}^{4}}+17 \frac{v_{3} y_{2}}{v_{1}^{4}}-68 \frac{v_{2} v_{3} y_{1}}{v_{1}^{5}}-105 \frac{v_{2}^{2} y_{2}}{v_{1}^{5}}+175 \frac{v_{2}^{3} y_{1}}{v_{1}^{6}}-\frac{y_{4}}{v_{1}^{3}}+3 \frac{v_{4} y_{1}}{v_{1}^{4}}\right) \\
& +6 r_{2}^{4}\left(N^{2}-1\right)\left(9 \frac{v_{2} y_{3}}{v_{1}^{4}}+9 \frac{v_{3} y_{2}}{v_{1}^{4}}-36 \frac{v_{2} v_{3} y_{1}}{v_{1}^{5}}\right. \\
& \left.\left.-36 \frac{v_{2}^{2} y_{2}}{v_{1}^{5}}+60 \frac{v_{2}^{3} y_{1}}{v_{1}^{6}}-\frac{y_{4}}{v_{1}^{3}}+3 \frac{v_{4} y_{1}}{v_{1}^{4}}\right)\right\}
\end{aligned}
$$

As before, we truncate by neglecting terms $\sim v_{4}$ and $\sim y_{4}$ and we further omit $\partial^{2} v_{1} / \partial \chi^{2}\left(\chi_{0}\right)$ and a term $\sim \frac{\partial^{3} U}{\partial \chi^{3}}\left(\chi_{0}\right) \frac{\partial \chi_{0}}{\partial t}$ in the flow equation for $m_{\chi}^{2}$ (4.14):

$$
\frac{\partial}{\partial t} m_{\chi}^{2}=\frac{9 \zeta(3)}{4 \pi^{2}}\left(N^{2}-1\right) k^{4} v_{1}^{-4} y_{1}^{2}
$$

We then end up with a closed system of differential equations for the eight functions $v_{1}, v_{2}, v_{3}, \chi_{0}, m_{\chi}^{2}, y_{1}, y_{2}, y_{3}$. This system can be solved numerically. The initial conditions for $y_{i}$ at the scale $k_{\chi}$ follow from (4.4):

$$
y_{1_{\mid k_{\chi}}}=-\frac{g^{2}\left(k_{n p}\right)}{\bar{g}^{2} a k_{\chi}}, \quad y_{2_{\mid k_{\chi}}}=0, \quad y_{3_{\mid k_{\chi}}}=0
$$


We note that the choice of $\bar{g}^{2}$ is arbitrary (it concerns only the overall normalization of $\theta$ ) and it seems convenient to take $\bar{g}^{2}=g^{2}\left(k_{n p}\right)$.

In order to make some quantitative comparison we compute in addition the trace of the energy momentum tensor $T_{\mu}^{\mu}$. In QCD its nonzero value is related to the running of the coupling constant and it is usually quoted as $T_{\mu}^{\mu}=-0.014 \mathrm{GeV}^{4}$. In our context the part of $T_{\mu}^{\mu}$ induced by the gluon condensate can be expressed in terms of the effective potential for $\chi$,

$$
T_{\mu}^{\mu}=4\left[U\left(\chi_{0}\right)-U(0)\right]
$$

Its value at $k=0$ can be computed from the flow equation (4.12)

$$
\frac{\partial}{\partial t} T_{\mu}^{\mu}=4 \frac{\partial}{\partial t} U_{k}\left(\chi_{0}\right)=\frac{3 \zeta(3)}{2 \pi^{2}}\left(N^{2}-1\right) k^{4}\left[v_{1}^{-2}-v_{1}(\chi=0)^{-2}\right]
$$

with $T_{\mu}^{\mu}\left(k_{\chi}\right)=0$. We use a linear approximation for $v_{1}(\chi)$, i.e. $v_{1}(\chi=0)=v_{1}-y_{1} \chi_{0}$. As an alternative, we can estimate $T_{\mu}^{\mu}$ in the approximation of a quadratic potential $U=\frac{1}{2} m_{\chi}^{2}\left(\chi-\chi_{0}\right)^{2}$, i.e.

$$
T_{\mu}^{\mu}=-2 m_{\chi}^{2} \chi_{0}^{2}
$$

We have solved the system of differential equations for $v_{1}, v_{2}, v_{3}, y_{1}, y_{2}, y_{3}, \chi_{0}$ and $m_{\chi}^{2}$ numerically. More precisely, we have first solved for $k>k_{n p}$ the system of flow equations of sect. 3 without composite fields. Here we have started at $k=4 \mathrm{GeV}$ with a gauge coupling adjusted in such a waythat the scale $k_{n p}$ coincides with a similarly defined scale in terms of the coupling in the $\overline{M S}$-scheme with three light quark flavours:

$$
\beta_{g^{2}} / g_{M S}^{2}\left(k_{n p}\right) \simeq-1.5
$$

In practice we set

$$
k_{n p}=2.11 \Lambda_{\mathrm{QCD}}^{\overline{M S}}=600 \mathrm{MeV}
$$

(for $\Lambda_{\mathrm{QCD}}^{\overline{M S}}=285 \mathrm{MeV}$ the two-loop confinement scale). At the scale $k_{n p}$ we also have introduced the composite field according to eq. (4.4), with $k_{\chi}=k_{n p}$. The results 
depend somewhat on the parameter $a$ that enters the initial values (4.15), (4.21), (4.27). We consider explicitly two values of $a$,

$$
a=-\frac{A}{\bar{g}^{2} w_{2}\left(k_{\chi}\right)}, \quad A=2(1.1)
$$

where the value in brackets corresponds to a situation for which the system becomes numerically unstable. For $A=3$ we obtain similar values as for $A=2$. We consider the values for $A=2$ as our best estimate and take the values for $A=1.1$ as an indication of the error.

One obtains for $k \rightarrow 0$ the same fixed point values for $v_{1}, v_{2}, v_{3}$ and $\alpha_{s}$ as found before without composite operators (end of the preceding section). This does not change for larger values of $a$. For $k=0$ the expectation value $\chi_{0}$ reaches the value

$$
\chi_{0}=0.65(1.45) \mathrm{GeV}
$$

for which

$$
<\chi>/ \Lambda_{\mathrm{QCD}}=2.3(5.1)
$$

and the " $F$ " condensate equals

$$
<F_{\mu \nu}^{z} F_{z}^{\mu \nu}>\equiv 4 k_{\chi}^{3}<\chi>=\left[3.1(3.7) \Lambda_{\mathrm{QCD}}\right]^{4}
$$

Here the first and the second figure always refers to $A=2$ and $A=1.1$, respectively. From the expressions (4.28), (4.30) for $T_{\mu}^{\mu}$ one finds

$$
T_{\mu}^{\mu}=-0.015(-0.039) \mathrm{GeV}^{4} \text { and } T_{\mu}^{\mu}=-0.0145(-0.0295) \mathrm{GeV}^{4} \text {, }
$$

respectively. Comparing with the value $T_{\mu}^{\mu}=-0.014 \mathrm{GeV}^{4}$ from $\mathrm{QCD}$ sum rules the agreement is almost perfect. Since we have made no attempt to define the renormalization scheme precisely and quarks are neglected, there is, however, a considerable scale ambiguity on top of the truncation errors. The mass term turns out as $m_{\chi}=130(83) \mathrm{MeV}$. This should, however, not yet be associated with the 
mass of a scalar glueball[" The latter needs knowledge about the kinetic term of the corresponding operator and could be defined as $m_{g b}=m_{\chi} Z_{\chi}^{-1 / 2}$. It seems possible to compute $Z_{\chi}$ from the solution of its flow equation, starting at $k_{\chi}$ with $Z_{\chi}=0$.

We do not think that the precise numbers should be taken too seriously. Even within our truncation one could solve the partial differential equation for $V_{k}(\theta, \chi)$ without a polynomial truncation. One may also investigate the dependence of the results on the parameters $k_{\chi}$ and $a$. This would also give a rough estimate of the truncation uncertainties since without truncations the final values of the condensates should be independent of $k_{\chi}$ and unique for a given definition of the composite operator. Nevertheless, the fact that expectation values come out in a reasonable order of magnitude seems to indicate that our approach offers a possible perspective for a quantitative understanding of the gluon condensate.

\section{Conclusions and outlook}

In this paper we have approximated the exact nonperturbative evolution equation for Yang-Mills theories [7] by a truncation where the effective action $\Gamma_{k}$ is given as a function $W_{k}$ of $\theta=\frac{1}{4} F_{\mu \nu} F^{\mu \nu}$. More precisely, we have investigated the effective action for constant magnetic fields $B$ in dependence on an infrared cutoff $k$ and solved the corresponding flow equation for $k \rightarrow 0$. Our ansatz is general enough to allow for a ground state value of $B$ different from zero. This would correspond to an absolute minimum of $W_{k}(\theta)$ for $\theta_{0}>0$ in the limit where the infrared cutoff $k$ vanishes. Polynomial approximations of $W_{k}$ in order $\theta$ and $\theta^{2}$ seem to indicate that the minimum of $W_{k \rightarrow 0}$ occurs for $\theta_{0}>0$, but are obviously insufficient since they lead to a function $W(\theta)$ which is not bounded from below. The minimal

\footnotetext{
${ }^{11}$ The quantity $m_{\chi}^{-1}$ should also not be confused with the correlation length for field strength fluctuations 23].
} 
realistic truncation in order $\theta^{3}$ leads to the conclusion that the minimum of $W_{k}(\theta)$ stabilizes at $\theta_{0}=0$ for all $k$. From an inspection of the general structure of the flow equation for $W_{k}(\theta)$ it seems likely that this feature is not an artifact of the polynomial approximation but rather a property of the partial differential equation for $W_{k}(\theta)$.

For a description of gluon condensation we have, in addition, introduced a gauge singlet field $\chi$ which is associated to a suitably regularized composite operator $F_{\mu \nu} F^{\mu \nu}$. Our computation of the effective potential for $\chi$ indicates a nonvanishing expectation value of $\chi$, related to a nonvanishing expectation value $<F_{\mu \nu} F^{\mu \nu}>\neq 0$. A first numerical investigation gives a value for the condensate $<F_{\mu \nu} F^{\mu \nu}>\approx$ $\left[3.1 \Lambda_{\mathrm{QCD}}\right]^{4}$ where $\Lambda_{\mathrm{QCD}}$ is the two-loop confinement scale in the $\overline{M S}$ scheme with three light flavors. The precise meaning of the operator $F_{\mu \nu} F^{\mu \nu}$ needs to be worked out - in the present version it roughly corresponds to $F_{\mu \nu} \hat{\theta}\left(k_{\chi}^{2}+D^{2}[A]\right) F^{\mu \nu}$ with $D^{2}$ the covariant Laplacian and $k_{\chi} \approx 2.1 \Lambda_{\mathrm{QCD}}$. Here $\hat{\theta}$ stands for a rather sharp cutoff (similar to the usual $\theta$-function) such that high eigenvalues of $-D^{2}$ do not contribute to the regularized operator. Even though we do not claim quantitative precision of our estimate we find it remarkable that the solution of relatively simple flow equations leads to a reasonable order of magnitude of the condensate!

For higher precision, and, in particular, for a possible comparison with more phenomenological approaches such as QCD sum rules or the stochastic QCD vacuum several shortcomings of the present truncation should be overcome. We list a few which seem to us particularly important:

(i) In the present truncation the momentum dependence of the inverse gluon propagator is always approximated by $Z_{k} q^{2}$, with $Z_{k}=W_{k}^{\prime}(0)$. For $k$ in the vicinity of $\Lambda_{\mathrm{QCD}}$ this is certainly not a very accurate approximation, even though the flow equations involve effectively only a small momentum range $q^{2} \approx k^{2}$ for a given scale 
$k$. A more reasonable approximation for the term quadratic in the gauge field $A_{\mu}$ would be of the sort

$$
\frac{1}{4} \int d^{4} x F_{\mu \nu}^{z} Z_{k}\left(-D^{2}[A]\right) F_{z}^{\mu \nu}
$$

with $Z_{k}$ depending on the covariant Laplacian in the adjoint representation ${ }^{12}$. For the functional form of $Z_{k}\left(q^{2}\right)$ we expect for large $q^{2}$ a $k$-independent positive function $Z\left(q^{2}\right)$. In lowest order the logarithmic dependence of $Z$ on $q^{2}$ should be determined by the one-loop $\beta$-function for the running gauge coupling. Indeed, external momenta of the gluons act as an independent infrared cutoff. For $k^{2} \ll q^{2}$ the running of $Z_{k}\left(q^{2}\right)$ with $k$ should essentially stop whereas it is given by the one-loop $\beta$-function for $k^{2} \gtrsim q^{2}$. In contrast, our truncation identifies $Z_{k}\left(q^{2}\right)$ with a constant $Z_{k}\left(q^{2}=0\right) \equiv W_{k}^{\prime}(\theta=0)$. In the truncations of sects. 3 and $4, W_{k}^{\prime}(\theta)$ remains positive for all $\theta$ such that the use of $Z_{k}=W_{k}^{\prime}(0)$ induces quantitative inaccuracy, but no qualitative problems.

ii) We observe that the spectrum of small fluctuations around the constant magnetic field configuration (2.3) lacks Euclidean $S O(4)$ rotation symmetry. This is not surprising since $F_{\mu \nu}$ singles out two of the space directions. In addition, the spectrum is partially discrete - continuity exists only with respect to the transversal momentum. The lack of full rotation symmetry and the partial discreteness of the spectrum are actually not unrelated: For an $S O(4)$ symmetric spectrum the continuity in two momentum directions must extend to all momentum directions. One

\footnotetext{
${ }^{12}$ The exact propagator will, in addition, also receive corrections from the modified gauge-fixing term $\hat{\Gamma}_{\text {gauge }}$ discussed in Appendix A.

${ }^{13}$ This would lead to unphysical problems for vanishing or negative $W_{k}^{\prime}$. With (5.1) the quantity $Z_{k}(0)$ could turn negative without affecting the high momentum behavior of $Z_{k}\left(q^{2}\right)$. With such a truncation the high momentum modes are always stable if the momentum dependence of the propagator is properly taken into account. This implies positive $u$ for $q^{2} \rightarrow \infty$ and no ultraviolet problem can appear for the exponential cutoff (1.2).
} 
expects then a spectrum with a few separate particles, each of them having $\Gamma_{k}^{(2)}$ depending continuously on a generalized $S O(4)$ invariant of the type $q_{\mu} q^{\mu}$. The lack of full rotation symmetry for the fluctuation spectrum in a given background is not a problem. Nevertheless, it would be interesting to check the validity of approximations by investigating background configurations with a different symmetry. In ref. [12] we have proposed a candidate for a configuration with generalized $S O(4)$ symmetry for $d=4$ and gauge group $S U(N), N \geq 4$. Unfortunately, no realistic candidate for the gauge group $S U(3)$ has been found up to now. It would be very interesting to perform an analysis similar to the one presented here for $S U(4)$, using the $S O(4)$ symmetric configuration instead of (2.3).

For the gauge group $S U(3)$ an interesting alternative configuration with constant colour-magnetic field is given by

$$
A_{i}^{z}=\left\{\begin{array}{ccc}
a \delta_{i}^{z} & \text { for } & i=1,2,3 \\
0 & \text { for } & i>3
\end{array}\right.
$$

(with $i=1,2,3$ corresponding to the spacelike indices in Minkowski space). It is invariant under the diagonal subgroup $S O(3)$ of three-dimensional space rotations and global gauge transformations (with generators $T_{1}, T_{2}, T_{3}$ forming an $S U(2)$ subgroup of $S U(3))$. The field strength corresponds to a constant colour-magnetic field

$$
F_{z i j}=\left\{\begin{array}{cl}
\bar{g} a^{2} \epsilon_{z i j} & \text { for } i, j=1,2,3 \\
0 & \text { otherwise }
\end{array}\right.
$$




$$
F_{z}^{\mu \nu} F_{\mu \nu}^{z}=2 B^{2}=6 \bar{g}^{2} a^{4}
$$

Nevertheless, $F_{\mu \nu}$ is not covariantly constant $(i, j, k=1,2,3)$

$$
\begin{gathered}
\left(F_{; k}^{i j}\right)_{z}=-\bar{g}^{2} a^{3}\left(\delta_{k}^{i} \delta_{z}^{j}-\delta_{k}^{j} \delta_{z}^{i}\right) \\
\left(-D^{2}[A]\right)^{y z} F_{i j}^{z}=2 \bar{g}^{2} a^{2} F_{i j}^{y}
\end{gathered}
$$

and this configuration allows to explore a nontrivial momentum dependence of the gluon propagator as in (5.1). For the characteristic properties of the spectrum of fluctuations around the configuration (5.2) it is sufficient to restrict the discussion to $d=3$. With the truncation $(2.1)$ we find that the spectrum is continuous. For large momenta it turns out positive semi-definite for all $B$, even for $k=0$. This contrasts with the spectrum for the background (2.3)! For a three-dimensional theory (high temperature field theory) the configuration (5.2) is an interesting ground state candidate. For the four-dimensional theory a study of $\Gamma_{k}$ as a function of $a$ should reveal additional aspects of the functional form of $\Gamma_{k}$.

In general, an investigation of the functional dependence of $\Gamma_{k}$ on various distinct configurations would greatly enhance the robustness of our results. Qualitative and quantitative results should be independent of the configuration chosen. The use of different configurations should therefore permit an estimate of the truncation uncertainties. In addition, different configurations project on different invariants and the use of several configurations would give much more detailed information about the full functional form of the effective action $\Gamma_{k}$.

iii) The present treatment of composite operators is still rather rough. One would prefer to work directly with a smooth definition of a regularized operator, as, for example,

$$
\chi \hat{=} \frac{1}{4 k_{\chi}^{3}} F_{\mu \nu} \exp \left(D^{2}[A] / k_{\chi}^{2}\right) F^{\mu \nu}
$$


This would permit a more direct comparison of the expectation value of $\chi$ with a corresponding weighted momentum integral of the two-point function for $F_{\mu \nu}$ and therefore with lattice results or phenomenological estimates. The general formalism for the treatment of this type of operators is known [13], but in practice it will require a more detailed study of the momentum dependence of $\Gamma_{k}$, beyond the truncation $\sim W_{k}(\theta)$. In fact, for the configuration with constant $B$ used in this paper $D^{2}[A] F^{\mu \nu}$ vanishes. At the present stage we can therefore not distinguish between different versions of a regularized $F_{\mu \nu} F^{\mu \nu}$ operator. (This is the reason why we cannot specify the function $\hat{\theta}$ mentioned above.) In order to resolve this issue, one needs an investigation of different configurations as described in ii).

In summary, our first attempt to investigate the phenomenon of gluon condensation with the help of nonperturbative flow equations is encouraging. The simple configuration (2.3) and the truncation (2.1) give a qualitatively interesting picture: Whereas the ground-state value of the gauge field $A_{\mu}$ vanishes, a composite operator $\sim F_{\mu \nu} F^{\mu \nu}$ develops a non-vanishing vacuum expectation value. For the moment our analysis is too rough for a detailed comparison with quantities that can be determined in lattice QCD simulations or by phenomenological estimates as QCD sum rules. We hope that a future extension of our investigation will allow for such a comparison. We find it remarkable that already a relatively simple scheme which starts from first principles and leads to a quantitative estimate of the gluon condensate.

Acknowledgement: We would like to thank D. Jungnickel for a first numerical solution of the differential equations of sect. 3 . 


\section{Appendix A}

In this appendix we derive the exact form of the evolution equation for $\Gamma_{k}$ as well as the modified Ward-Takahashi or Slavnov-Taylor identities and "background field identities" which it satisfies. We start from the scale dependent generating functional

$$
\begin{aligned}
\exp W_{k}\left[K_{\mu}^{z}, \sigma^{z}, \bar{\sigma}^{z} ; \bar{\beta}_{\mu}^{z}, \bar{\gamma}^{z} ; \bar{A}_{\mu}^{z}\right] & =\int \mathcal{D} \mathcal{A D C D} \bar{C} \exp -\left\{S[\mathcal{A}]+\Delta_{k} S\right. \\
\left.+S_{\text {gf }}+S_{\text {ghost }}+S_{\text {source }}\right\} & =\int \mathcal{D} \phi \exp -S_{\text {tot }}
\end{aligned}
$$

where $S[\mathcal{A}]$ denotes the gauge invariant classical action, and

$$
\begin{aligned}
\Delta_{k} S= & \frac{1}{2} \int d^{d} x(\mathcal{A}-\bar{A})_{\mu}^{y} R_{k}(\bar{A})_{\mu \nu}^{y z}(\mathcal{A}-\bar{A})_{\nu}^{z} \\
& +\int d^{d} x \bar{C}^{y} R_{k}(\bar{A})^{y z} C^{z}
\end{aligned}
$$

is the infrared cutoff for the gauge field fluctuation $a \equiv \mathcal{A}-\bar{A}$ and for the FaddeevPopov ghosts $C$ and $\bar{C}$. Here $R_{k}(\bar{A})$ is a suitable cutoff operator which depends on $\bar{A}$ only. It may be chosen differently for the gauge field and for the ghosts. Furthermore

$$
S_{\mathrm{gf}}=\frac{1}{2 \alpha} \int d^{d} x\left[D_{\mu}(\bar{A})^{y z}(\mathcal{A}-\bar{A})_{\mu}^{z}\right]^{2}
$$

is the background gauge fixing term and

$$
S_{\text {ghost }}=-\int d^{d} x \bar{C}^{y}\left(D_{\mu}(\bar{A}) D_{\mu}(\mathcal{A})\right)^{y z} C^{z}
$$

is the corresponding ghost action [16]. The fields $\mathcal{A}-\bar{A}, \bar{C}$ and $C$ are coupled to the sources $K, \sigma$ and $\bar{\sigma}$, respectively:

$$
\begin{aligned}
S_{\text {source }}= & -\int d^{d} x\left\{K_{\mu}^{z}\left(\mathcal{A}_{\mu}^{z}-\bar{A}_{\mu}^{z}\right)+\bar{\sigma}^{z} C^{z}+\sigma^{z} \bar{C}^{z}\right. \\
& \left.+\frac{1}{g} \bar{\beta}_{\mu}^{y} D_{\mu}(\mathcal{A})^{y z} C^{z}+\frac{1}{2} \bar{\gamma}^{w} f^{w y z} C^{y} C^{z}\right\} .
\end{aligned}
$$

(In this appendix we use throughout $g$ instead of $\bar{g}$.) We also included the sources $\bar{\beta}$ and $\bar{\gamma}$ which couple to the BRS-variations of $\mathcal{A}$ and of $C$, respectively. In fact, 
$S+S_{g f}+S_{\text {ghost }}$ is invariant under the BRS transformation

$$
\begin{aligned}
\delta \mathcal{A}_{\mu}^{w} & =\frac{1}{g} \varepsilon D_{\mu}(\mathcal{A})^{w z} C^{z} \\
\delta C^{w} & =-\frac{1}{2} \varepsilon f^{w y z} C^{y} C^{z} \\
\delta \bar{C}^{w} & =\frac{\varepsilon}{\alpha g} D_{\mu}(\bar{A})^{w z}\left(\mathcal{A}_{\mu}^{z}-\bar{A}_{\mu}^{z}\right) .
\end{aligned}
$$

Let us introduce the classical fields

$$
\bar{a}_{\mu}^{z}=\frac{\delta W_{k}}{\delta K_{\mu}^{z}}, \xi^{z}=\frac{\delta W_{k}}{\delta \bar{\sigma}^{z}}, \bar{\xi}^{z}=\frac{\delta W_{k}}{\delta \sigma^{z}}
$$

and let us formally solve the relations $\bar{a}=\bar{a}(K, \sigma, \bar{\sigma} ; \bar{\beta}, \bar{\gamma} ; \bar{A}), \xi=\xi(\ldots)$, etc., for the sources $K, \sigma$ and $\bar{\sigma}: K=K(\bar{a}, \xi, \bar{\xi} ; \bar{\beta}, \bar{\gamma} ; \bar{A}), \sigma=\sigma(\ldots), \ldots$ We introduce the new functional $\tilde{\Gamma}_{k}$ as the Legendre transform of $W_{k}$ with respect to $K, \sigma$ and $\bar{\sigma}$ :

$$
\begin{aligned}
\tilde{\Gamma}_{k}[\bar{a}, \xi, \bar{\xi} ; \bar{\beta}, \bar{\gamma} ; \bar{A}]= & \int d^{d} x\left\{K_{\mu}^{z} \bar{a}_{\mu}^{z}+\bar{\sigma}^{z} \xi^{z}+\sigma^{z} \bar{\xi}^{z}\right\} \\
& -W_{k}[K, \sigma, \bar{\sigma} ; \bar{\beta}, \bar{\gamma} ; \bar{A}] .
\end{aligned}
$$

Apart from the usual relations

$$
\frac{\delta \tilde{\Gamma}_{k}}{\delta \bar{a}_{\mu}^{z}}=K_{\mu}^{z}, \quad \frac{\delta \tilde{\Gamma}_{k}}{\delta \xi^{z}}=-\bar{\sigma}^{z}, \quad \frac{\delta \tilde{\Gamma}_{k}}{\delta \bar{\xi}^{z}}=-\sigma^{z}
$$

we have also

$$
\frac{\delta \tilde{\Gamma}_{k}}{\delta \bar{\beta}_{\mu}^{z}}=-\frac{\delta W_{k}}{\delta \bar{\beta}_{\mu}^{z}}, \quad \frac{\delta \tilde{\Gamma}_{k}}{\delta \bar{\gamma}^{z}}=-\frac{\delta W_{k}}{\delta \bar{\gamma}^{z}}
$$

where $\delta \tilde{\Gamma} / \delta \bar{\beta}$ is taken for fixed $\bar{a}, \xi, \bar{\xi}$ and $\delta W / \delta \bar{\beta}$ for fixed $K, \sigma, \bar{\sigma}$ etc.

The effective average action $\Gamma_{k}$ is obtained by subtracting the IR cutoff $\Delta_{k} S$, expressed in terms of the classical fields, from the Legendre transform $\tilde{\Gamma}_{k}$ :

$$
\begin{aligned}
\Gamma_{k}[\bar{a}, \xi, \bar{\xi} ; \bar{\beta}, \bar{\gamma} ; \bar{A}]= & \tilde{\Gamma}_{k}[\bar{a}, \xi, \bar{\xi} ; \bar{\beta}, \bar{\gamma} ; \bar{A}]-\frac{1}{2} \int d^{d} x \bar{a}_{\mu}^{y} R_{k}(\bar{A})_{\mu \nu}^{y z} \bar{a}_{\nu}^{z} \\
& -\int d^{d} x \bar{\xi}^{y} R_{k}(\bar{A})^{y z} \xi^{z}
\end{aligned}
$$


Frequently we shall use the field $A \equiv \bar{A}+\bar{a}$ (the classical counterpart of $\mathcal{A} \equiv \bar{A}+a$ ) and write correspondingly

$$
\Gamma_{k}[A, \bar{A}, \xi, \bar{\xi} ; \bar{\beta}, \bar{\gamma}] \equiv \Gamma_{k}[A-\bar{A}, \xi, \bar{\xi} ; \bar{\beta}, \bar{\gamma} ; \bar{A}] .
$$

For $\xi=\bar{\xi}=\bar{\beta}=\bar{\gamma}=0$ one recovers the effective average action $\Gamma_{k}[A, \bar{A}]$ introduced in ref. [7]. Using the methods described there one finds the following exact evolution equation governing its scale-dependence, with $t=\ln k$

$$
\begin{aligned}
& \frac{\partial}{\partial t} \Gamma_{k}[A, \bar{A}, \xi, \bar{\xi} ; \bar{\beta}, \bar{\gamma}]=\frac{1}{2} \operatorname{Tr}_{x c L}\left[\left(\Gamma_{k}^{(2)}+R_{k}(\bar{A})\right)_{A A}^{-1} \frac{\partial}{\partial t} R_{k}(\bar{A})_{A A}\right] \\
& -\frac{1}{2} \operatorname{Tr}_{x c}\left[\left(\left(\Gamma_{k}^{(2)}+R_{k}(\bar{A})\right)\right)_{\bar{\xi} \xi}^{-1}-\left(\Gamma_{k}^{(2)}+R_{k}(\bar{A})\right)_{\xi \bar{\xi}}^{-1} \frac{\partial}{\partial t} R_{k}(\bar{A})_{\bar{\xi} \xi}\right] .
\end{aligned}
$$

Here $\Gamma_{k}^{(2)}$ is the Hessian of $\Gamma_{k}$ with respect to $A, \xi$ and $\bar{\xi}$ at fixed $\bar{A}, \bar{\beta}$ and $\bar{\gamma}$ and $R_{k A A}, R_{k \bar{\xi} \xi}$ are the infrared cutoffs introduced in (A.2). (We use for the ghosts the convention $\left.\left(\Gamma_{\bar{\xi} \xi}^{(2)}\right)^{y z}=\frac{\delta^{2} \Gamma}{\delta \xi^{z} \delta \xi^{y}},\left(\Gamma_{\xi \bar{\xi}}^{(2)}\right)^{y z}=\frac{\delta^{2} \Gamma}{\delta \xi^{z} \delta \xi^{y}}.\right)$

It is clear from its construction that $\Gamma_{k}$ is invariant under simultaneous gauge transformations of $A_{\mu}$ and $\bar{A}_{\mu}$ and homogeneous transformations of $\xi, \bar{\xi}, \bar{\beta}_{\mu}$ and $\bar{\gamma}$, i.e., $\delta \Gamma_{k}[A, \bar{A}, \xi, \bar{\xi} ; \bar{\beta}, \bar{\gamma}]=0$ for

$$
\begin{aligned}
\delta A_{\mu}^{y} & =-\frac{1}{g} D_{\mu}(A)^{y z} \omega^{z} \\
\delta \bar{A}_{\mu}^{y} & =-\frac{1}{g} D_{\mu}(\bar{A})^{y z} \omega^{z} \\
\delta V^{y} & =f^{y w z} V^{w} \omega^{z}, V \equiv \xi, \bar{\xi}, \bar{\beta}_{\mu}, \bar{\gamma} .
\end{aligned}
$$

Next we turn to the Ward identities. By applying the transformations (A.6) to the integrand of (A.1) one obtains from the BRS invariance of the measure $\mathcal{D} \phi$

$$
\int \mathcal{D} \phi \delta_{B R S} \exp -S_{\text {tot }}=0
$$


or

$$
\begin{aligned}
& \int d^{d} x\left\{K_{\mu}^{z} \frac{\delta W_{k}}{\delta \bar{\beta}_{\mu}^{z}}+\bar{\sigma}^{z} \frac{\delta W_{k}}{\delta \bar{\gamma}^{z}}-\frac{1}{\alpha g} \sigma^{y} D_{\mu}(\bar{A})^{y z} \frac{\delta W_{k}}{\delta K_{\mu}^{z}}\right\} \\
= & \int d^{d} x\left\{\left[\frac{\delta W_{k}}{\delta \bar{\beta}_{\mu}^{z}}+\frac{\delta}{\delta \bar{\beta}_{\mu}^{z}}\right]\left(R_{k} \frac{\delta W_{k}}{\delta K}\right)_{\mu}^{z}+\frac{1}{\alpha g}\left(D_{\mu}(\bar{A})\left[\frac{\delta W_{k}}{\delta K_{\mu}}+\frac{\delta}{\delta K_{\mu}}\right]\right)^{z}\left(R_{k} \frac{\delta W_{k}}{\delta \bar{\sigma}}\right)^{z}\right. \\
+ & {\left.\left[\frac{\delta W_{k}}{\delta \sigma^{z}}+\frac{\delta}{\delta \sigma^{z}}\right]\left(R_{k} \frac{\delta W_{k}}{\delta \bar{\gamma}}\right)^{z}\right\} }
\end{aligned}
$$

with $\left(R_{k} \delta W_{k} / \delta \bar{\gamma}\right)^{z} \equiv R_{k}(\bar{A})^{z y} \delta W_{k} / \delta \bar{\gamma}^{y}$, etc. Equation (A.16) can be converted to the following relation for the effective average action (

$$
\int d^{d} x\left\{\frac{\delta \Gamma_{k}^{\prime}}{\delta A_{\mu}^{z}} \frac{\delta \Gamma_{k}^{\prime}}{\delta \bar{\beta}_{\mu}^{z}}-\frac{\delta \Gamma_{k}^{\prime}}{\delta \xi^{z}} \frac{\delta \Gamma_{k}^{\prime}}{\delta \bar{\gamma}^{z}}\right\}=\Delta_{k}^{(B R S)}
$$

where the symmetry-breaking contribution $\Delta_{k}^{(\mathrm{BRS})}$ is given by

$$
\begin{aligned}
\Delta_{k}^{(B R S)=} & \operatorname{Tr}_{x c L}\left[R_{k}(\bar{A})_{A_{\mu} A_{\nu}}\left(\Gamma_{k}^{(2)}+R_{k}\right)_{A_{\mu} \varphi}^{-1} \frac{\delta^{2} \Gamma_{k}^{\prime}}{\delta \varphi \delta \bar{\beta}_{\nu}}\right] \\
& -\operatorname{Tr}_{x c}\left[R_{k}(\bar{A})_{\bar{\xi} \xi}\left(\Gamma_{k}^{(2)}+R_{k}\right)_{\xi \varphi}^{-1} \frac{\delta^{2} \Gamma_{k}^{\prime}}{\delta \varphi \delta \bar{\gamma}}\right] \\
& -\frac{1}{\alpha g} \operatorname{Tr}_{x c}\left[D_{\mu}(\bar{A})\left(\Gamma_{k}^{(2)}+R_{k}\right)_{A_{\mu} \bar{\xi}}^{-1} R_{k}(\bar{A})_{\bar{\xi} \xi}\right]
\end{aligned}
$$

and where

$$
\Gamma_{k}^{\prime} \equiv \Gamma_{k}-\frac{1}{2 \alpha} \int d^{d} x\left[D_{\mu}(\bar{A})\left(A_{\mu}-\bar{A}_{\mu}\right)\right]^{2} .
$$

Here $\varphi \equiv\left(A_{\mu}, \xi, \bar{\xi}\right)$ is summed over on the r.h.s. of (A.18) and the traces on the r.h.s. of (A.18) involve an $x$ integration as well as a sum over the suppressed index $z$. In deriving eq. (A.18) we used

$$
\left[\frac{\delta}{\delta \bar{\xi}^{y}}-g D_{\mu}(\bar{A})^{y z} \frac{\delta}{\delta \bar{\beta}_{\mu}^{z}}\right] \Gamma_{k}[A, \bar{A}, \xi, \bar{\xi} ; \bar{\beta}, \bar{\gamma}]=0
$$

which follows from the equation of motion of the antighost.

Equation (A.17) is the generating relation for the modified Ward identities which we wanted to derive. In conventional Yang-Mills theory, without IR-cutoff, the r.h.s. of (A.17) is zero. The traces on the r.h.s. of (A.17) lead to a violation of the usual 
Ward identities for nonvanishing values of $k$. As $k$ approaches zero, $R_{k}$ and hence $\Delta_{k}^{(\mathrm{BRS})}$ vanishes and we recover the conventional Ward-Takahashi identities. Eq. (A.17) is equivalent to a similar identity derived in ref. [11] using a different gauge.

The modified Ward identities A.17) are not the only conditions which the average action $\Gamma_{k}$ has to satisfy. Next we derive a relation which constrains its dependence on the background gauge field $\bar{A}_{\mu}$. Taking the $\bar{A}$-derivative of (A.1) one obtains

$$
\begin{aligned}
& -\frac{\delta W_{k}}{\delta \bar{A}_{\mu}^{z}(y)}=-\left(R_{k} \bar{a}\right)_{\mu}^{z}(y)+\frac{1}{\alpha}(\bar{D} \otimes \bar{D} \bar{a})_{\mu}^{z}(y)+K_{\mu}^{z}(y) \\
& +\int d^{d} x\left\{\frac{1}{2} \bar{a}_{\nu} \frac{\delta\left(R_{k}-\frac{1}{\alpha} \bar{D} \otimes \bar{D}\right)_{\nu \tau}}{\delta \bar{A}_{\mu}^{z}(y)} \bar{a}_{\tau}+\bar{\xi} \frac{\delta R_{k}}{\delta \bar{A}_{\mu}^{z}(y)} \xi\right\} \\
& +\frac{1}{2} \operatorname{Tr}_{x c L}\left[\frac{\delta^{2} W_{k}}{\delta K_{\tau} \delta K_{\nu}} \frac{\delta}{\delta \bar{A}_{\mu}^{z}(y)}\left(R_{k}-\frac{1}{\alpha} \bar{D} \otimes \bar{D}\right)_{\nu \tau}\right] \\
& -\operatorname{Tr}_{x c}\left[\frac{\delta^{2} W_{k}}{\delta \bar{\sigma} \delta \sigma} \frac{\delta}{\delta \bar{A}_{\mu}^{z}(y)} R_{k}\right] \\
& -g^{2} \bar{\xi}^{v}(y) f^{v z w} \frac{\delta W_{k}}{\delta \bar{\beta}_{\mu}^{w}(y)}+g^{2} f^{z v w} \frac{\delta^{2} W_{k}}{\delta \sigma^{v}(y) \delta \bar{\beta}_{\mu}^{w}(y)}
\end{aligned}
$$

with $(\bar{D} \otimes \bar{D})_{\mu \nu}^{v w} \equiv D_{\mu}(\bar{A})^{v z} D_{\nu}(\bar{A})^{z w}$. Upon Legendre transforming eq. (A.21) and switching from $\tilde{\Gamma}_{k}$ to $\Gamma_{k}$ one arrives at the following result

$$
\begin{aligned}
& \frac{\delta}{\delta \bar{A}_{\mu}^{z}(y)} \Gamma_{k}^{\prime}[A, \bar{A}, \xi, \bar{\xi} ; \bar{\beta}, \bar{\gamma}]=-g^{2} \bar{\xi}^{v}(y) f^{z v w} \frac{\delta \Gamma_{k}}{\delta \bar{\beta}_{\mu}^{w}(y)} \\
& +\frac{1}{2} \operatorname{Tr}_{x c L}\left[\left(\Gamma_{k}^{(2)}+R_{k}\right)_{A A}^{-1} \frac{\delta}{\delta \bar{A}_{\mu}^{z}(y)}\left(R_{k}-\frac{1}{\alpha} \bar{D} \otimes \bar{D}\right)_{A A}\right] \\
& -\operatorname{Tr}_{x c}\left[\left(\Gamma_{k}^{(2)}+R_{k}\right)_{\bar{\xi} \xi}^{-1} \frac{\delta R_{k \bar{\xi} \xi}}{\delta \bar{A}_{\mu}^{z}(y)}\right] \\
& +g^{2} \int d^{d} x \operatorname{tr}_{c}\left[T^{z}\left(\Gamma_{k}^{(2)}+R_{k}\right)_{\bar{\xi}(y) \varphi(x)}^{-1} \frac{\delta^{2} \Gamma_{k}}{\delta \varphi(x) \delta \bar{\beta}_{\mu}(y)}\right]
\end{aligned}
$$

Again, $\varphi \equiv(A, \xi, \bar{\xi})$ is summed over and $\left(T^{z}\right)^{y w}=-i f^{z y w}$. Note that the r.h.s. of eq. (A.22) does not vanish even for $k \rightarrow 0$. The $\bar{D} \otimes \bar{D}$-piece of the 2 nd term and the 4th term on the r.h.s. of (A.22) survive this limit. 
So far we were deriving general identities which constrain the form of the exact functional $\Gamma_{k}$. Let us now ask what they imply if we truncate the space of actions. In the present paper we neglect the $k$-evolution of the ghost sector by making an ansatz which keeps the classical form of the corresponding terms in the action:

$$
\begin{aligned}
& \Gamma_{k}[A, \bar{A}, \xi, \bar{\xi} ; \bar{\beta}, \bar{\gamma}]=\Gamma_{k}[A, \bar{A}]+\Gamma_{\mathrm{gh}} \\
\Gamma_{\mathrm{gh}}= & -\int d^{d} x \bar{\xi} D_{\mu}(\bar{A}) D_{\mu}(A) \xi \\
& -\int d^{d} x\left\{\frac{1}{g} \bar{\beta}_{\mu}^{v}\left(D_{\mu}(A)^{v w} \xi^{w}+\frac{1}{2} \bar{\gamma}^{u} f^{u v w} \xi^{v} \xi^{w}\right\}\right.
\end{aligned}
$$

If we insert this truncation into the general evolution equation (A.13), we obtain precisely eq. (1.1), which is our starting point in the main body of the paper. A generic functional $\Gamma_{k}[A, \bar{A}]$ can be decomposed according to

$$
\Gamma_{k}[A, \bar{A}]=\bar{\Gamma}_{k}[A]+\frac{1}{2 \alpha} \int d^{d} x\left[D_{\mu}(\bar{A})\left(A_{\mu}-\bar{A}_{\mu}\right)\right]^{2}+\hat{\Gamma}_{k}^{\text {gauge }}[A, \bar{A}]
$$

where $\bar{\Gamma}_{k}$ is defined by equating the two-gauge fields: $\bar{\Gamma}_{k}[A] \equiv \Gamma_{k}[A, A]$. The remainder $\Gamma_{k}[A, \bar{A}]-\bar{\Gamma}_{k}[A]$ is further decomposed in the classical gauge-fixing term plus a correction to it, $\hat{\Gamma}_{k}^{\text {gauge }}$, which also contains the counterterms. Note that $\hat{\Gamma}_{k}^{\text {gauge }}[A, A]=0$ for equal gauge and background fields. We observe that $\bar{\Gamma}_{k}[A]$ is a gauge-invariant functional of $A_{\mu}$ and $\Gamma_{k}[A, \bar{A}]$ is invariant under a simultaneous gauge transformation of $A$ and $\bar{A}$.

In the present paper we make the further approximation of neglecting quantum corrections to the gauge fixing term and $k$-dependent counterterms by setting $\hat{\Gamma}_{k}^{\text {gauge }}=0$. Then (A.25) coincides with eq. (2.1) for

$$
\bar{\Gamma}_{k}[A]=\int d^{d} x W_{k}\left(\frac{1}{4} F^{2}\right) .
$$

The important question is whether this truncation is consistent with the WardTakahashi identities (A.17) and the $\bar{A}$-derivative (A.22), respectively. If we insert 
(A.23)-(A.25) into A.17), for instance, we find that $\bar{\Gamma}_{k}$ drops out from the l.h.s. of this equation. We are left with a condition for $\hat{\Gamma}_{k}^{\text {gauge }}$

$$
-\frac{1}{g} \int d^{d} x \frac{\delta \hat{\Gamma}^{\text {gauge }}}{\delta \bar{A}_{\mu}^{z}(x)}\left(D_{\mu}(A) \xi\right)^{z}(x)=\Delta_{k}^{(\mathrm{BRS})}
$$

The term $\Delta_{k}^{(\mathrm{BRS})}$ (A.18) vanishes for $k \rightarrow 0$ but is non-zero for $k>0$. Our approximation $\hat{\Gamma}_{k}^{\text {gauge }} \equiv 0$ is consistent provided these terms can be neglected. We note that the traces appearing in (A.27) are related to higher loop effects. Beyond a loop approximation our neglection of $\hat{\Gamma}^{\text {gauge }}$ is a non-trivial assumption. We emphasize that because of its gauge invariance the functional $\bar{\Gamma}_{k}[A]$ does not appear on the l.h.s. of the Ward identities. Therefore the Ward identities do not imply any further condition for $\bar{\Gamma}_{k}$. This means that, within the approximations made, we may write down any ansatz for $\bar{\Gamma}_{k}$ as long as it is gauge-invariant.

Similar remarks apply to the identity for the $\bar{A}$-dependence (A.22). If we insert (A.23) with (A.25) into (A.22) the first term on the r.h.s. is cancelled by $\delta \Gamma_{\mathrm{gh}} / \delta \bar{A}_{\mu}^{z}(y)$. One obtains

$$
\begin{aligned}
\frac{\delta \hat{\Gamma}_{k}^{\text {gauge }}}{\delta \bar{A}_{\mu}^{z}(y)}= & -\frac{1}{2 \alpha} \operatorname{Tr}_{x c L}\left[\left(\Gamma_{k}^{(2)}+R_{k}\right)_{A A}^{-1} \frac{\delta \bar{D} \otimes \bar{D}}{\delta \bar{A}_{\mu}^{z}(y)}\right] \\
& +g^{2} \int d^{d} x \operatorname{tr}_{c}\left[T^{z}\left(\Gamma_{k}^{(2)}+R_{k}\right)_{\bar{\xi}(y) \varphi(x)}^{-1} \frac{\delta^{2} \Gamma_{k}}{\delta \varphi(x) \delta \bar{\beta}_{\mu}(y)}\right] \\
& +\operatorname{Tr}\left[R_{k}(\ldots)\right]
\end{aligned}
$$

Consistency of the truncation $\hat{\Gamma}_{k}^{\text {gauge }}=0$ requires that we neglect the traces on the r.h.s. of (A.28). Contrary to the case of the Ward identities, not all of these terms vanish obviously for $k \rightarrow 0$. We observe again that, within the present approximation, the equation for the $\bar{A}$-dependence does not impose any restriction on $\bar{\Gamma}_{k}$.

We finally observe that the flow equation (1.1) can be rewritten in close analogy 
to a one-loop formula:

$$
\begin{aligned}
\frac{\partial}{\partial t} \Gamma_{k}[A, \bar{A}]= & \frac{1}{2} \frac{D}{D t} \operatorname{Tr}_{x c L} \ln \left[\Gamma_{k}^{(2)}[A, \bar{A}]+R_{k}\left(\Gamma_{k}^{(2)}[\bar{A}, \bar{A}]\right)\right] \\
& -\frac{D}{D t} \operatorname{Tr}_{x c} \ln \left[-D^{\mu}[A] D_{\mu}[\bar{A}]+R_{k}\left(-D^{2}[\bar{A}]\right)\right]
\end{aligned}
$$

The derivative $\frac{D}{D t}$ acts only on the explicit $k$-dependence of the function $R_{k}$, but not on $\Gamma_{k}^{(2)}[A, \bar{A}]$. It is now easy to describe the relation between the effective average action $\Gamma_{k}$ and the conventional perturbative effective action. Let us first briefly discuss the approximation $\frac{D}{D t} \rightarrow \frac{\partial}{\partial t}$ in eq. (1.3). This amounts to neglecting the running of $\Gamma_{k}$ on the r.h.s. of the evolution equation. It is then trivial to solve it explicitly:

$$
\begin{aligned}
\Gamma_{k}[A, \bar{A}]= & \Gamma_{\Lambda}[A, \bar{A}]+\frac{1}{2} \operatorname{Tr}_{x c L}\left\{\ln \left[\Gamma_{k}^{(2)}[A, \bar{A}]+R_{k}\left(\Gamma_{k}^{(2)}[\bar{A}, \bar{A}]\right)\right]\right. \\
& \left.-\ln \left[\Gamma_{\Lambda}^{(2)}[A, \bar{A}]+R_{\Lambda}\left(\Gamma_{\Lambda}^{(2)}[\bar{A}, \bar{A}]\right)\right]\right\} \\
& -\operatorname{Tr}_{x c}\left\{\ln \left[-D^{\mu}[A] D_{\mu}[\bar{A}]+R_{k}\left(-D^{2}[\bar{A}]\right)\right]\right. \\
& \left.-\ln \left[-D^{\mu}[A] D_{\mu}[\bar{A}]+R_{\Lambda}\left(-D^{2}[\bar{A}]\right)\right]\right\}+O\left(\frac{\partial}{\partial t} \Gamma_{k}^{(2)}\right)
\end{aligned}
$$

with $\Lambda$ some appropriate high momentum scale (ultraviolet cutoff) where we may identify $\Gamma_{\Lambda}$ with the classical action $S$ including a gauge fixing term and counterterms. This formula has a similar structure as a regularized expression for the conventional one-loop effective action in the background gauge [16, 2]. There are two important differences, however:

(i) The second variation of the classical action, $S^{(2)}$, is replaced by $\Gamma_{k}^{(2)}$. This implements a kind of "renormalization group improvement" and transforms (A.29) into a sort of "gap equation".

(ii) The effective average action contains an explicit infrared cutoff $R_{k}$. For the choice (1.2) one has

$$
\lim _{u \rightarrow \infty} R_{k}(u)=0, \quad \lim _{u \rightarrow 0} R_{k}(u)=Z_{k} k^{2} .
$$


Effectively, a $k$-dependent mass-type term is added to the inverse propagator $\Gamma_{k}^{(2)}$ for the low frequency modes $(u \rightarrow 0)$, but it is absent for the high frequency modes $(u \rightarrow \infty)$. Despite the similarity of $(1.3)$ with a one-loop expression, we stress that, for $k \rightarrow 0$, the solution of the original renormalization group equation where $D / D t$ does not act on $\Gamma_{k}^{(2)}$ equals the exact effective action which includes contributions from all orders of the loop expansion ${ }^{14}$.

For a detailed discussion of the approximation (A.29) in the case of the abelian Higgs model we refer to [14, and to ref. 15] for the corresponding nonperturbative evolution equations of this model.

\section{Appendix B}

In this appendix we discuss the technical steps needed for the derivation of the truncated flow equation (2.5) from the exact equation (A.13). Upon performing the second variation of the ansatz (2.1),

$$
\delta^{2} \Gamma_{k}[A, \bar{A}]=\int d^{d} x \delta A_{y}^{\mu} \Gamma_{k}^{(2)}[A, \bar{A}]_{\mu \nu}^{y z} \delta A_{z}^{\nu}
$$

we arrive at the following operator $\Gamma_{k}^{(2)}$ :

$$
\Gamma_{k}^{(2)}[A, \bar{A}]_{\mu \nu}^{y z}=W_{k}^{\prime}(\theta)\left(\mathcal{D}_{T}[A]-\mathcal{D}_{L}[A]\right)_{\mu \nu}^{y z}+W_{k}^{\prime \prime}(\theta) \mathcal{S}_{\mu \nu}^{y z}[A]+\frac{1}{\alpha_{k}}\left(\mathcal{D}_{L}[\bar{A}]\right)_{\mu \nu}^{y z}
$$

with

$$
\theta=\frac{1}{4} F_{\mu \nu}^{z} F_{z}^{\mu \nu}
$$

Here we used the notation $(w, y, z$ are adjoint group indices and $\bar{g}$ is the (bare) gauge coupling)

$$
\left(\mathcal{D}_{T}\right)_{\mu \nu}^{y z}=\left(-D^{2} \delta_{\mu \nu}+2 i \bar{g} F_{\mu \nu}\right)^{y z}
$$

\footnotetext{
${ }^{14}$ This holds for the exact flow equation (A.13), whereas (1.1) involves already an approximation in the ghost sector.
} 


$$
\begin{aligned}
\left(\mathcal{D}_{L}\right)_{\mu \nu}^{y z} & =-(D \otimes D)_{\mu \nu}^{y z}=-D_{\mu}^{y w} D_{\nu}^{w z} \\
\mathcal{S}_{\mu \nu}^{y z} & =F_{\mu \rho}^{y} F_{\sigma \nu}^{w}\left(D^{\rho} D^{\sigma}\right)^{w z}
\end{aligned}
$$

with the covariant derivative $\left(D_{\mu}[A]\right)^{y w}=\partial_{\mu} \delta^{y w}-i \bar{g} A_{\mu}^{z}\left(T_{z}\right)^{y w}$ in the adjoint representation and $F_{\mu \nu}^{y w}=F_{\mu \nu}^{z}\left(T_{z}\right)^{y w}$. Moreover, $W_{k}^{\prime}$ and $W_{k}^{\prime \prime}$ denote the first and the second derivative of $W_{k}$ with respect to $\theta$.

In writing down eq. (B.4) we made the additional assumption that the field strength $F_{\mu \nu}[A]$ is covariantly constant $F_{\mu \nu ; \rho}^{z}=0$ or

$$
\left[D_{\rho}[A], F_{\mu \nu}[A]\right]=0
$$

It is easy to see that (2.3) and (2.4) obey the condition (B.5). The choice (2.3) has the advantage that it allows for an explicit diagonalization of the operator $\Gamma_{k}^{(2)}$.

We note that $W_{k}$ can be extracted from $\Gamma_{k}[A, A]$ which is a gauge-invariant functional of $A$ obtained by putting $\bar{A}=A$. It is therefore sufficient to know $\Gamma_{k}^{(2)}[A, A]$.

Before turning to the evolution equation, we list a few special properties of the covariantly constant fields, which will prove helpful later on. From (2.3) it follows that $A_{\mu}$ satisfies the classical Yang-Mills equations $D^{\mu} F_{\mu \nu}=0$. This in turn is sufficient to prove that the operators $\mathcal{D}_{T}$ and $\mathcal{D}_{L}$ commute. As a consequence, one may define generalized projection operators [7]

$$
\begin{aligned}
& P_{L}=\mathcal{D}_{T}^{-1} \mathcal{D}_{L} \\
& P_{T}=1-P_{L}
\end{aligned}
$$

which satisfy $P_{T, L}^{2}=P_{T, L}, P_{T}+P_{L}=1$ and $P_{T} P_{L}=0=P_{L} P_{T}$. For $A_{\mu}=0$ they reduce to the standard projectors on transverse and longitudinal modes:

$$
\begin{aligned}
& \left(P_{T}^{(0)}\right)_{\mu \nu}=\delta_{\mu \nu}-\partial_{\mu} \partial_{\nu} / \partial^{2} \\
& \left(P_{L}^{(0)}\right)_{\mu \nu}=\partial_{\mu} \partial_{\nu} / \partial^{2}
\end{aligned}
$$


Furthermore, if $A_{\mu}$ is of the form (2.3), it is natural to define another pair of orthogonal projectors,

$$
P_{\perp}^{y z}=\delta^{y z}-n^{y} n^{z}, \quad P_{\|}^{y z}=n^{y} n^{z}
$$

which project on the spaces perpendicular and parallel to $n^{z}$, respectively. For the vector potential (2.3) the matrix $A_{\mu}^{w} T_{w}$ reads in the adjoint representation

$$
A_{\mu}^{y z}(x) \equiv\left(A_{\mu}^{w} T_{w}\right)^{y z}=i f^{y w z} n_{w} \mathrm{~A}_{\mu}(x)
$$

The antisymmetry of the structure constants $f^{y w z}$ implies that $P_{\|}$and $P_{\perp}$ commute with $D_{\mu}, D^{2}, \mathcal{D}_{T}, \mathcal{D}_{L}$ and $F_{\mu \nu}$, and that

$$
P_{\|} A_{\mu}=0, \quad P_{\|} D_{\mu}=P_{\|} \partial_{\mu}, \quad P_{\|}\left(\mathcal{D}_{T}\right)_{\mu \nu}=-\partial^{2} \delta_{\mu \nu} P_{\|}
$$

The operator $\mathcal{S}$ from (B.4) factorizes according to

$$
\begin{aligned}
\mathcal{S}_{\mu \nu}^{y z} & =P_{\|}^{y z} s_{\mu \nu}, \\
s_{\mu \nu} & =\mathrm{F}_{\mu \rho} \mathrm{F}_{\sigma \nu} \partial^{\rho} \partial^{\sigma}
\end{aligned}
$$

Hence $\mathcal{S}$ commutes with $D^{2}, \mathcal{D}_{L}$ and $\mathcal{D}_{T}$ because it annihilates the gauge-field contained in these operators:

$$
\mathcal{S} D^{2}=\mathcal{S} \partial^{2}, \quad \mathcal{S} \mathcal{D}_{T}=-\mathcal{S} \partial^{2}, \quad \mathcal{S} D \otimes D=\mathcal{S} \partial \otimes \partial
$$

In physical terms this means that those components of the gauge fluctuations $\delta A_{\mu}^{z} \equiv a_{\mu}^{z}$ which are parallel to $n^{z}$ decouple from $A_{\mu}$ to some extent. In fact, in terms of the projections $a_{\mu}^{\perp, \|}=P_{\perp, \|} a_{\mu}$ the quadratic action (B.1) with (B.5) reads

$$
\begin{aligned}
& \delta^{2} \Gamma_{k}[A, A]=\int d^{d} x\left\{a_{z}^{\| \mu}\left[-\partial^{2} W_{k}^{\prime} \delta_{\mu \nu}+\left(W_{k}^{\prime}-\frac{1}{\alpha_{k}}\right) \partial_{\mu} \partial_{\nu}+W_{k}^{\prime \prime} s_{\mu \nu}\right] a^{\| \nu z}\right. \\
& \left.+a_{y}^{\perp \mu}\left[W_{k}^{\prime} \mathcal{D}_{T}+\left(\frac{1}{\alpha_{k}}-W_{k}^{\prime}\right) \mathcal{D}_{L}\right]_{\mu \nu}^{y z} a_{z}^{\perp \nu}\right\}
\end{aligned}
$$

We observe that the $a^{\|}$-modes couple to the external field only via the derivatives of $W_{k} \equiv W_{k}\left(\frac{1}{2} B^{2}\right)$. In a conventional one-loop calculation one uses the classical YangMills Lagrangian $\frac{1}{4} F_{\mu \nu}^{2}$ rather than $W_{k}\left(\frac{1}{4} F_{\mu \nu}^{2}\right)$. In that case the quadratic action for 
the small fluctuations is given by $(\underline{B} .13)$ with $W_{k}^{\prime}=1$ and $W_{k}^{\prime \prime}=0$. Hence the oneloop determinant resulting from the integration over $a^{\|}$is field-independent and may be ignored. In the present case, the $a^{\|}$-modes are important for the "renormalization group improvement", however.

The quadratic form $(\mathbb{B} .13)$ can be diagonalized even further by introducing the longitudinal and transversal projections

$$
\begin{aligned}
& a^{\|, L}=P_{L} a^{\|}=P_{L}^{(0)} a^{\|}, \quad a^{\|, T}=P_{T} a^{\|}=P_{T}^{(0)} a^{\|} \\
& a^{\perp, L}=P_{L} a^{\perp}, \quad a^{\perp, T}=P_{T} a^{\perp}
\end{aligned}
$$

By virtue of $P_{L}^{(0)} s=s P_{L}^{(0)}=0, P_{T}^{(0)} s=s P_{T}^{(0)}=s$ and $\left[P_{L(T)}, \theta\right]=0$ one obtains

$$
\begin{aligned}
\delta^{2} \Gamma_{k}[A, A]= & \int d^{d} x\left\{a_{z}^{\|, T, \mu}\left[-\partial^{2} W_{k}^{\prime} \delta_{\mu \nu}+W_{k}^{\prime \prime} s_{\mu \nu}\right] a^{\|, T, \nu, z}\right. \\
& +\frac{1}{\alpha_{k}} a_{z}^{\|, L, \mu}\left[-\partial^{2}\right] a_{\mu}^{\|, L, z} \\
& +a_{y}^{\perp, T, \mu}\left[W_{k}^{\prime} \mathcal{D}_{T}\right]_{\mu \nu}^{y z} a_{z}^{\perp, T, \nu} \\
& \left.+\frac{1}{\alpha_{k}} a_{y}^{\perp, L, \mu}\left[\mathcal{D}_{T}\right]_{\mu \nu}^{y z} a_{z}^{\perp, L, \nu}\right\}
\end{aligned}
$$

This block-diagonal form of $\Gamma_{k}^{(2)}$ will facilitate the evolution of the traces occurring in the evaluation equation. For example, $a^{\|, L}$ gives no $A$-dependent contribution and, except for an irrelevant constant, the only dependence of $\Gamma_{k}$ on $\alpha_{k}$ arises from $a^{\perp, L}$. Writing

$$
\Gamma^{(2)}[A, A]=\Gamma_{1}^{(2)}+\Gamma_{2}^{(2)}+\Gamma_{3}^{(2)}+\Gamma_{4}^{(2)}
$$

where, in an obvious notation

$$
\begin{aligned}
& \Gamma_{1}^{(2)}=P_{\|} P_{T} \Gamma_{\|, T}^{(2)} P_{\|} P_{T}, \quad \Gamma_{2}^{(2)}=P_{\|} P_{L} \Gamma_{\|, L}^{(2)} P_{\|} P_{L} \\
& \Gamma_{3}^{(2)}=P_{\perp} P_{T} \Gamma_{\perp, T}^{(2)} P_{\perp} P_{T}, \quad \Gamma_{4}^{(2)}=P_{\perp} P_{L} \Gamma_{\perp, L}^{(2)} P_{\perp} P_{L}
\end{aligned}
$$

with $\left[P_{\|, \perp}, P_{L, T}\right]=0$ and

$$
\begin{aligned}
& \Gamma_{A}^{(2)} \Gamma_{B}^{(2)}=0 \text { for } A \neq B \\
& {\left[\Gamma_{A}^{(2)}, \Gamma_{B}^{(2)}\right]=0}
\end{aligned}
$$


one obtains $\left(\left[\Gamma_{\|, T}^{(2)}, P_{\|} P_{T}\right]=0\right.$ etc. $)$

$$
\begin{aligned}
R_{k}\left(\Gamma^{(2)}\right)= & P_{\|} P_{T} R_{k}\left(\Gamma_{\|, T}^{(2)}\right) P_{\|} P_{T}+P_{\|} P_{L} R_{k}\left(\Gamma_{\|, L}^{(2)}\right) P_{\|} P_{L} \\
& +P_{\perp} P_{T} R_{k}\left(\Gamma_{\perp, T}^{(2)}\right) P_{\perp} P_{T}+P_{\perp} P_{L} R_{k}\left(\Gamma_{\perp, L}^{(2)}\right) P_{\perp} P_{L}
\end{aligned}
$$

We will choose the matrix $\mathcal{Z}_{k}$ in the definition of $R_{k}(1.2)$ as $\mathcal{Z}_{k}=1$ for the ghosts and

$$
\mathcal{Z}_{k}=Z_{k} P_{T}[\bar{A}]+\tilde{Z}_{k} P_{L}[\bar{A}]
$$

for the gauge boson degrees of freedom. Here $Z_{k}, \tilde{Z}_{k}$ are $k$-dependent constants and we observe that the choice $(\mathbb{B} .20)$ is compatible with $(\mathbb{B} .19)$. If we insert the truncation (2.1) into (1.1) with $\bar{A}=A$, we obtain $\left(\theta=\frac{1}{2} B^{2}\right)$

$$
\begin{aligned}
\Omega \frac{\partial}{\partial t} W_{k}(\theta)= & \frac{1}{2} \operatorname{Tr}_{x c L}\left[H\left(\Gamma_{k}^{(2)}[A, A]\right)\right] \\
& -\operatorname{Tr}_{x c}\left[H_{G}\left(-D^{2}[A]\right)\right] \\
& +\frac{1}{2} \operatorname{Tr}_{x c L}\left[P_{\perp} P_{L}\left(\tilde{H}\left(\Gamma_{\perp L}^{(2)}\right)-H\left(\Gamma_{\perp L}^{(2)}\right)\right)\right]
\end{aligned}
$$

where $\Gamma_{k}^{(2)}$ is given by $(\mathbb{B} .5), H(u)$ is defined by eq. (2.7) and $\Omega \equiv \int d^{d} x$.

Let us pause here for a moment and derive a set of trace identities which will be needed for the evaluation of (B.21). For the covariantly constant background (2.3), eq. (B.10) implies for any function $f$

$$
\operatorname{Tr}_{x c L}\left[P_{\|} f\left(D_{\mu}, P_{\|}, P_{\perp}\right)\right]=\operatorname{Tr}_{x L}\left[f\left(\partial_{\mu}, 1,0\right)\right]
$$

because $\operatorname{Tr}_{c}\left[P_{\|}\right]=n^{z} n_{z}=1$. Writing $P_{\perp}=1-P_{\|}$and exploiting $\mathcal{S} \propto P_{\|}$it is also easy to see that

$$
\operatorname{Tr}_{x c L}\left[P_{\perp} f\left(D_{\mu}, \mathcal{S}\right)\right]=\operatorname{Tr}_{x c L}\left[f\left(D_{\mu}, 0\right)\right]-\operatorname{Tr}_{x L}\left[f\left(\partial_{\mu}, 0\right)\right]
$$

Since $\mathrm{F}_{\mu \nu}$ is a constant matrix, the operator $s_{\mu \nu}$ of (B.11) commutes with $P_{L}^{(0)}$ and $P_{T}^{(0)}$ and satisfies $\partial^{\mu} s_{\mu \nu}=0$. This fact can be used to show that

$$
\operatorname{Tr}_{x L}\left[f\left(P_{L}^{(0)}, P_{T}^{(0)} ; s\right)\right]
$$




$$
=\operatorname{Tr}_{x L}[f(0,1 ; s)]+\operatorname{Tr}_{x}[f(1,0 ; 0)]-\operatorname{Tr}_{x}[f(0,1 ; 0)]
$$

If one subtracts the same expression with $s=0$ one obtains

$$
\begin{aligned}
& \operatorname{Tr}_{x L}\left[f\left(P_{L}^{(0)}, P_{T}^{(0)} ; s\right)-f\left(P_{L}^{(0)}, P_{T}^{(0)} ; 0\right)\right] \\
= & \operatorname{Tr}_{x L}[f(0,1 ; s)]-d \operatorname{Tr}_{x}[f(0,1 ; 0)]
\end{aligned}
$$

In the last step we used that $\operatorname{Tr}_{x L}=d \operatorname{Tr}_{x}$ for an operator $\sim \delta_{\mu \nu}$. In the above identities the function $f$ may also depend on further operators provided they commute with those displayed explicitly and do not introduce any additional colour or Lorentz index structures.

For the evaluation of $U_{1}$ in eq. (B.32) we need another important relation:

$$
\operatorname{Tr}_{x c L}\left[P_{L} f\left(\mathcal{D}_{T}\right)\right]=\operatorname{Tr}_{x c}\left[f\left(-D^{2}\right)\right]
$$

It follows from the fact that the operator $\left(\mathcal{D}_{T}\right)_{\mu \nu}=-D^{2} \delta_{\mu \nu}+2 i \bar{g} F_{\mu \nu}$, when restricted to the space of longitudinal modes $\left(a_{\mu}=\left(P_{L}\right)_{\mu}^{\nu} a_{\nu}\right)$, has the same spectrum as $-D^{2}$ acting on Lorentz scalars. The proof makes essential use of the identity

$$
\mathcal{D}_{T}(D \otimes D)=(D \otimes D) \mathcal{D}_{T}=-(D \otimes D)(D \otimes D)
$$

which holds true whenever the gauge field contained in the covariant derivatives obeys $D^{\mu} F_{\mu \nu}=0$.

Equipped with the above trace identities, we now resume the evaluation of the flow equation $(\mathbb{B . 2 1})$. The first trace on the r.h.s. of eq. (B.21) can be simplified as follows. Inserting a factor of $1=P_{\|}+P_{\perp}$ leads to the decomposition

$$
\operatorname{Tr}_{x c L}\left[H\left(\Gamma_{k}^{(2)}[A, A]\right)\right]=T_{1}^{\|}+T_{1}^{\perp}
$$

with

$$
\begin{aligned}
T_{1}^{\|} & =\operatorname{Tr}_{x c L}\left[P_{\|} H\left(W_{k}^{\prime} \mathcal{D}_{T}+\left[W_{k}^{\prime}-\frac{1}{\alpha_{k}}\right] D \otimes D+W_{k}^{\prime \prime} \mathcal{S}\right)\right] \\
& =\operatorname{Tr}_{x L}\left[H\left(-W_{k}^{\prime} \partial^{2}+\left[W_{k}^{\prime}-\frac{1}{\alpha_{k}}\right] \partial \otimes \partial+W_{k}^{\prime \prime} s\right]\right.
\end{aligned}
$$


where $(\mathbb{B . 2 2})$ was used, and

$$
\begin{aligned}
T_{1}^{\perp}= & \operatorname{Tr}_{x c L}\left[P_{\perp} H\left(W_{k}^{\prime} \mathcal{D}_{T}+\left[W_{k}^{\prime}-\frac{1}{\alpha_{k}}\right] D \otimes D+W_{k}^{\prime \prime} \mathcal{S}\right)\right] \\
= & \operatorname{Tr}_{x c L}\left[H\left(W_{k}^{\prime} \mathcal{D}_{T}+\left[W_{k}^{\prime}-\frac{1}{\alpha_{k}}\right] D \otimes D\right)\right] \\
& -\operatorname{Tr}_{x L}\left[H\left(-W_{k}^{\prime} \partial^{2}+\left[W_{k}^{\prime}-\frac{1}{\alpha_{k}}\right] \partial \otimes \partial\right)\right]
\end{aligned}
$$

where $(\overline{B .23})$ was exploited. Let us write

$$
\operatorname{Tr}_{x c L}\left[H\left(\Gamma_{k}^{(2)}[A, A]\right)\right]=U_{1}+U_{2}
$$

with $U_{1}$ the "nonabelian" trace

$$
U_{1}=\operatorname{Tr}_{x c L}\left[H\left(W_{k}^{\prime} \mathcal{D}_{T}+\left[W_{k}^{\prime}-\frac{1}{\alpha_{k}}\right] D \otimes D\right)\right]
$$

and $U_{2}$ the sum of $T_{1}^{\|}$and the second term of $(\overline{B .30})$ :

$$
\begin{aligned}
U_{2}= & \operatorname{Tr}_{x L}\left[H\left(-\partial^{2}\left[W_{k}^{\prime} P_{T}^{(0)}+\frac{1}{\alpha_{k}} P_{L}^{(0)}\right]+W_{k}^{\prime \prime} s\right)\right] \\
& -\operatorname{Tr}_{x L}\left[H\left(-\partial^{2}\left[W_{k}^{\prime} P_{T}^{(0)}+\frac{1}{\alpha_{k}} P_{L}^{(0)}\right]\right)\right]
\end{aligned}
$$

It is quite remarkable that if we now apply the identity (B.25) to $U_{2}$, the longitudinal contribution drops out completely and the result becomes independent of the gauge fixing parameter $\alpha_{k}$ :

$$
U_{2}=\operatorname{Tr}_{x L}\left[H\left(-\partial^{2} W_{k}^{\prime}+W_{k}^{\prime \prime} s\right)\right]-d \operatorname{Tr}_{x}\left[H\left(-\partial^{2} W_{k}^{\prime}\right)\right]
$$

The operators entering (B.34) are easily diagonalized in a plane-wave basis. A standard calculation yields, for $W_{k}^{\prime}>0, W_{k}^{\prime}+B^{2} W_{k}^{\prime \prime}>0$,

$$
\Omega^{-1} U_{2}=2 v_{d}\left(\frac{1}{W_{k}^{\prime}+B^{2} W_{k}^{\prime \prime}}-\frac{1}{W_{k}^{\prime}}\right)\left(\frac{1}{W_{k}^{\prime}}\right)^{\frac{d}{2}-1} \int_{0}^{\infty} d x x^{\frac{d}{2}-1} H(x)
$$

with $v_{d}=\left[2^{d+1} \pi^{d / 2} \Gamma(d / 2)\right]^{-1}$. (As always, the argument of $W_{k}$ and its derivatives is understood to be $\frac{1}{2} B^{2}$.) 
Next let us simplify the trace $U_{1}$ by inserting a pair of projectors:

$$
\begin{aligned}
U_{1} & =\operatorname{Tr}_{x c L}\left[P_{T} H\left(\mathcal{D}_{T}\left[W_{k}^{\prime} P_{T}+\frac{1}{\alpha_{k}} P_{L}\right]\right)\right]+\operatorname{Tr}_{x c L}\left[P_{L} H\left(\mathcal{D}_{T}\left[W_{k}^{\prime} P_{T}+\frac{1}{\alpha_{k}} P_{L}\right]\right)\right] \\
& =\operatorname{Tr}_{x c L}\left[P_{T} H\left(W_{k}^{\prime} \mathcal{D}_{T}\right)\right]+\operatorname{Tr}_{x c L}\left[P_{L} H\left(\frac{1}{\alpha_{k}} \mathcal{D}_{T}\right)\right] \\
& =\operatorname{Tr}_{x c L}\left[H\left(W_{k}^{\prime} \mathcal{D}_{T}\right)\right]+\triangle U_{1}\left(\alpha_{k}\right) .
\end{aligned}
$$

The $\alpha$-dependence of $U_{1}$ is contained in

$$
\begin{aligned}
\triangle U_{1}\left(\alpha_{k}\right) & =\operatorname{Tr}_{x c L}\left[P_{L}\left\{H\left(\frac{1}{\alpha_{k}} \mathcal{D}_{T}\right)-H\left(W_{k}^{\prime} \mathcal{D}_{T}\right)\right\}\right] \\
& =\operatorname{Tr}_{x c}\left[H\left(-\frac{1}{\alpha_{k}} D^{2}\right)-H\left(-W_{k}^{\prime} D^{2}\right)\right] .
\end{aligned}
$$

In the last line of (B.37) we made use of the identity (B.26).

By a similar combination of the trace identities we can also evaluate the last term on the r.h.s. of (B.21)

$$
\begin{aligned}
& \operatorname{Tr}_{x c L}\left\{P_{\perp} P_{L}\left(\tilde{H}\left(\frac{\mathcal{D}_{T}}{\alpha_{k}}\right)-H\left(\frac{\mathcal{D}_{T}}{\alpha_{k}}\right)\right)\right\} \\
= & \operatorname{Tr}_{x c}\left\{\tilde{H}\left(-\frac{D^{2}}{\alpha_{k}}\right)-H\left(-\frac{D^{2}}{\alpha_{k}}\right)\right\} \\
& -\operatorname{Tr}_{x}\left\{\tilde{H}\left(-\frac{\partial^{2}}{\alpha_{k}}\right)-H\left(-\frac{\partial^{2}}{\alpha_{k}}\right)\right\} .
\end{aligned}
$$

Evaluating the second term in a plane wave basis yields

$$
\frac{1}{2 \Omega} \operatorname{Tr}_{x}\left\{\tilde{H}\left(-\frac{\partial^{2}}{\alpha_{k}}\right)-H\left(-\frac{\partial^{2}}{\alpha_{k}}\right)\right\}=v_{d} \int_{0}^{\infty} d x x^{\frac{d}{2}-1}\left(\tilde{H}\left(\frac{x}{\alpha_{k}}\right)-H\left(\frac{x}{\alpha_{k}}\right)\right)
$$

At this point we have exploited the various trace identities as much as possible.

Combining these results yields the flow equation (2.5).

\section{Appendix C}

In this appendix we discuss the group theoretical factors $\sum_{\ell} \nu_{\ell}^{2 m}$ appearing in the Euler-McLaurin expansion of the spectral sums. 
The LHS of the evolution equation is $\partial_{t} W_{k}$. The argument of $W_{k}$ is $\frac{1}{4} F_{\mu \nu}^{z} F_{z}^{\mu \nu}=$ $\frac{1}{2} B^{2}$ which is manifestly independent of the unit vector $n^{z}$ which specifies the direction of the field in "color space". The r.h.s. of the evolution equation consists of expansions such as (2.11) which involve the factors $\sum_{\ell} \nu_{\ell}^{2 m}$. As $\left\{\nu_{\ell}\right\}$ are the eigenvalues of $n^{z} T_{z}$, we can rewrite them as

$$
\sum_{\ell} \nu_{\ell}^{2 m}=n^{z_{1}} n^{z_{2}} \cdots n^{z_{2 m}} \operatorname{Tr}_{c}\left[T_{z_{1}} T_{z_{2}} \cdots T_{z_{2 m}}\right]
$$

where the trace is in the adjoint representation. The question is whether the invariants (C.1) are all independent of the direction of $n^{z}$. In appendix D we explain in detail that generically this is not the case. If the orbit space of the gauge group in the adjoint representation is nontrivial, different $n$ 's can lead to different sums $\sum_{\ell} \nu_{\ell}^{2 m}$. The resolution to this puzzle is as follows. For $m=1$ we can use the standard orthogonality relation

$$
\operatorname{Tr}_{c}\left[T_{y} T_{z}\right]=N \delta_{y z}
$$

to prove that $\sum_{\ell} \nu_{\ell}^{2}=N n^{z} n_{z}=N$ is independent of the direction of $n$. Likewise, if the symmetric invariant tensor $\operatorname{Tr}_{c}\left[T_{\left(z_{1}\right.} \cdots T_{\left.z_{2 m}\right)}\right]$ is proportional to the trivial one, $\delta_{\left(z_{1} z_{2}\right.} \delta_{z_{3} z_{4}} \cdots \delta_{\left.z_{2 m-1} z_{2 m}\right)}$, we can again use the normalization condition $n^{z} n_{z}=1$ to show that the r.h.s. of (C.1) is independent of $n$.

The situation changes if there exists a totally symmetric invariant tensor $\mathcal{T}_{z_{1} z_{2} \cdots z_{2 m}}$ which is different from the trivial one. Then we might have

$$
\operatorname{Tr}_{c}\left[T_{\left(z_{1}\right.} \cdots T_{\left.z_{2 m}\right)}\right]=\tau_{m} \delta_{\left(z_{1} z_{2}\right.} \cdots \delta_{\left.z_{2 m-1} z_{2 m}\right)}+\mathcal{T}_{z_{1} z_{2} \cdots z_{2 m}}
$$

with some coefficient $\tau_{m}$. (If there exists more than one $\mathcal{T}$ an appropriate sum is implied.) In general $n^{z_{1}} n^{z_{2}} \ldots \mathcal{T}_{z_{1} z_{2} \ldots}$ will be direction dependent [25]. If some invariant tensor $\mathcal{T}$ exists, the correct way of deriving the evolution equation for $W_{k}$ is to compare coefficients of a fixed tensor structure on both sides of the equation. Clearly 
the l.h.s., $\partial_{t} W_{k}\left(\frac{1}{4} F_{\mu \nu}^{2}\right)$, gives rise to the trivial tensor structure only. Therefore only the $\tau_{m}$-piece of (C.3) should be kept in (C.1) and the ( $n$-dependent) part coming from $\mathcal{T}$ has to be discarded. Thus (2.11) may be used on the r.h.s. of the equation for $W_{k}$ provided we interpret $\sum \nu_{l}^{2 m}$ as the coefficient $\tau_{m}$.

On the other hand, a nontrivial $\mathcal{T}$ permits us to construct additional invariants from an even number of covariantly conserved $F_{\mu \nu}$. Then the truncation $W(\theta)$ is not sufficient any more to parametrize the most general effective action for constant magnetic fields of the type introduced in sect. 2 (with covariantly constant $F_{\mu \nu}$ ). The evolution equation for the new invariants can now be extracted by projecting the r.h.s. on the appropriate tensor structure. We will not pursue this generalization in the present paper.

In appendix D we show that for $S U(2)$ this complication is absent. There exists no additional invariant tensor $\mathcal{T}$, and one finds the $n$-independent result

$$
\sum_{l=1}^{3} \nu_{l}^{2 m}=2
$$

for all $m=1,2 \ldots$

\section{Appendix D}

In this appendix we investigate in more detail the group-theoretical quantities $\sum_{l} \nu_{l}^{2 m}$ which occur in many calculations involving covariantly constant backgrounds of the type $A_{\mu}^{z}=n^{z} \mathrm{~A}_{\mu}$. Here we consider an arbitrary (semi-simple, compact) gauge group $G$ with structure constants $f^{w y z}$. For a fixed unit vector $n^{z}$ we consider the matrix

$$
\hat{n}^{y z}=n^{w}\left(T^{w}\right)^{y z}=i f^{y w z} n^{w}
$$

The numbers $\nu_{l}, l=1, \ldots, \operatorname{dim} G$ are the eigenvalues of $\hat{n}: \hat{n}^{y z} \psi_{l}^{z}=\nu_{l} \psi_{l}^{y}$. This equation can be rewritten in a more suggestive form. Let $t^{z}$ denote the generators 
of $G$ in an arbitrary representation: $\left[t^{w}, t^{y}\right]=i f^{w y z} t^{z}$. If we define

$$
\tilde{n}=n^{z} t^{z}, \quad \tilde{\psi}_{l}=\psi_{l}^{z} t^{z}
$$

the eigenvalue equation becomes

$$
\left[\tilde{n}, \tilde{\psi}_{l}\right]=\nu_{l} \tilde{\psi}_{l}
$$

Clearly the $\nu_{l}$ 's do not depend on the representation chosen. We would like to know how the spectrum $\left\{\nu_{l}\right\}$ depends on the vector $n$. First of all, it is clear that if $V$ is any group element in the $t$-representation, the matrices $\tilde{n}$ and $\tilde{n}^{\prime}=V \tilde{n} V^{-1}$ have the same spectrum, i.e. the spectrum is constant along the orbit of $G$ in the adjoint representation. If two directions $n$ and $n^{\prime}$ are not related by a group transformation, then the spectra $\left\{\nu_{l}(n)\right\}$ and $\left\{\nu_{l}\left(n^{\prime}\right)\right\}$ can be different. Typically, for $G$ large enough [25], the orbit space is indeed nontrivial, and the spectrum "feels" the direction of $n^{z}$.

Let us go over from the basis $\left\{T^{z}\right\}$ to the Cartan-Weyl basis $\left\{H_{i}, E_{\vec{\alpha}}\right\}$ of the abstract Lie algebra. Here $\vec{\alpha} \in \mathbb{R}^{r}$ are the root vectors and $i=1, \ldots, r \equiv \operatorname{rank} G$. For definiteness we assume that the $t^{z}$ 's are in the fundamental representation where we write $\left\{h_{i}, e_{\vec{\alpha}}\right\}$ for the Cartan-Weyl basis. Thus

$$
\left[H_{i}, E_{\vec{\alpha}}\right]=\alpha_{i} E_{\vec{\alpha}} \quad \text { and } \quad\left[h_{i}, e_{\vec{\alpha}}\right]=\alpha_{i} e_{\vec{\alpha}}
$$

We assume that the generators $h_{i}$ of the Cartan subalgebra are given by diagonal matrices. By an appropriate transformation $\tilde{n} \rightarrow V \tilde{n} V^{-1}$ any $\tilde{n}$ can be brought to diagonal form. Therefore, in order to investigate the $n$-dependence of $\left\{\nu_{l}(n)\right\}$, it is sufficient to consider $\tilde{n}$ 's which are in the Cartan subalgebra: $\tilde{n}=\sum_{i=1}^{r} n_{i} h_{i}$. For this choice

$$
\left[\tilde{n}, e_{\vec{\alpha}}\right]=\left(\sum_{i=1}^{r} n_{i} \alpha_{i}\right) e_{\vec{\alpha}}, \quad\left[\tilde{n}, h_{i}\right]=0
$$


and the nonvanishing eigenvalues $\nu_{l}=\nu_{\vec{\alpha}}$ are given by

$$
\nu_{\vec{\alpha}}=\sum_{i=1}^{r} n_{i} \alpha_{i}
$$

Therefore the quantities $\sum_{l} \nu_{l}^{2 m}$ can be computed explicitly from the root system:

$$
\sum_{l=1}^{\operatorname{dim} G} \nu_{l}^{2 m}=\sum_{\text {roots }\{\vec{\alpha}\}}\left(\sum_{i=1}^{\text {rank } G} n_{i} \alpha_{i}\right)^{2 m}
$$

Let us consider a few simple examples. For $G=S U(2)$ we have $r=1, n_{1}=1$ and there are only two (one-component) roots: $\alpha= \pm 1$. Thus

$$
\sum_{l=1}^{3} \nu_{l}^{2 m}=2, \quad m=1,2,3, \ldots
$$

depends neither on the direction $n^{z}$ nor on the power $m$. This degeneracy can be understood by noting that for $S U(2)$ the square of the matrix (D.1) is the projector $P_{\perp}: \hat{n} \hat{n}=P_{\perp}$. This means that $\sum_{l} \nu_{l}^{2 m}=\operatorname{Tr}\left(P_{\perp}^{m}\right)=\operatorname{Tr}\left(P_{\perp}\right)=2$, as it should be. Contracting eq. (C.3) with $n^{z_{1}} \ldots n^{z_{2 m}}$ and comparing the result to (D.8) we see that there exists no nontrivial invariant tensor $\mathcal{T}$ and that $\tau_{m}=2$ for all $m$.

For $G=S U(3)$ we have $r=2$, and a 2-component unit vector $\left(n_{1}, n_{2}\right)$ specifies the direction of the field in the Cartan subalgebra. Using the explicit form of the roots it is straightforward to derive that

$$
\sum_{l=1}^{8} \nu_{l}^{2 m}=2^{1-2 m}\left[\left(n_{1}+\sqrt{3} n_{2}\right)^{2 m}+\left(n_{1}-\sqrt{3} n_{2}\right)^{2 m}+\left(2 n_{1}\right)^{2 m}\right]
$$

For $m=1$ and $m=2$ it turns out that this expression depends on $n_{1}$ and $n_{2}$ only via $n_{1}^{2}+n_{2}^{2}=1$, and one obtains the direction-independent results

$$
\sum_{l=1}^{8} \nu_{l}^{2}=3, \quad \sum_{l=1}^{8} \nu_{l}^{4}=\frac{9}{4}
$$

Starting from $m=3$, the invariants are explicitly $n$-dependent. Writing $n_{1}=$ $\cos \theta, n_{2}=\sin \theta$ we find for $m=3$

$$
\sum_{l=1}^{8} \nu_{l}^{6}=\frac{3}{16}\left[11 \cos ^{6} \theta+15 \cos ^{4} \theta \sin ^{2} \theta+45 \cos ^{2} \theta \sin ^{4} \theta+9 \sin ^{6} \theta\right]
$$


As discussed in Appendix $\mathrm{C}$, the $n$-dependence is related to the existence of a nontrivial invariant tensor $\mathcal{T}_{z_{1} \ldots z_{6}}$. However, we are not going to calculate the corresponding coefficient $\tau_{3}$ here. On the other side we note that the definition of $\tau$ in (C.3) depends on the specific truncation. Different truncations - i.e. different choices of the definition of a term $\sim \mathcal{T}_{z_{1} \ldots z_{6}} F_{\mu \nu}^{z_{1}} F_{\mu \nu}^{z_{2}} F_{\rho \sigma}^{z_{3}} F_{\rho \sigma}^{z_{4}} F_{\tau \lambda}^{z_{5}} F_{\tau \lambda}^{z_{6}}$ whose coefficient is set to zero - correspond to a different angle $\theta$ for which the r.h.s. of (D.11) equals $\tau_{3}$. For $N=3$ we may therefore use $\tau_{2}=\frac{9}{4}$ and $\tau_{3}$ between $\frac{27}{16}$ and $\frac{33}{16}$, the last uncertainty reflecting the uncertainty from this particular part of the truncation.

\section{Appendix E}

In the regime where $\bar{g} B / k^{2} \gtrsim 1$ the use of the Euler-McLaurin expansion (2.11) becomes questionable and we should look for an alternative representation of the spectral sums (2.9) and (2.10). In this section we use the Schwinger proper-time representation [20]. It can be easily applied only for the simplified cutoff function

$$
R_{k}(x)=Z_{k} k^{2} .
$$

In this case one may write $\left(x \equiv \mathcal{D}_{T}\right)$

$$
H(x) \equiv \frac{\partial_{t} R_{k}(x)}{x+R_{k}(x)}=\frac{\partial}{\partial t}\left(Z_{k} k^{2}\right) \int_{0}^{\infty} d s e^{-s Z_{k} k^{2}} e^{-s x}
$$

Inserting this representation into (2.9) we may employ

$$
\sum_{n=0}^{\infty} \exp \left[-s W_{k}^{\prime} \bar{g}\left|\nu_{l}\right| B(2 n+1)\right]=\frac{1}{2 \sinh \left[s W_{k}^{\prime} \bar{g}\left|\nu_{l}\right| B\right]}
$$

and

$$
\int_{0}^{\infty} d x x^{\frac{d}{2}-2} e^{-s W_{k}^{\prime} x}=v_{d-2}^{-1} 2^{1-d} \pi^{1-\frac{d}{2}}\left(s W_{k}^{\prime}\right)^{1-\frac{d}{2}}
$$

as long as $W_{k}^{\prime}>0$. One finds

$$
\Omega^{-1} \operatorname{Tr}_{x c L}\left[H\left(W_{k}^{\prime} \mathcal{D}_{T}\right)\right]=2(4 \pi)^{-\frac{d}{2}}\left(W_{k}^{\prime}\right)^{1-\frac{d}{2}}
$$




$$
\begin{aligned}
& \cdot \frac{\partial}{\partial t}\left(Z_{k} k^{2}\right) \sum_{l=1}^{N^{2}-1} \bar{g}\left|\nu_{l}\right| B \int_{0}^{\infty} \frac{d s}{s} s^{(4-d) / 2} e^{-s Z_{k} k^{2}} \\
& \cdot\left[\frac{d}{2 \sinh \left(s W_{k}^{\prime} \bar{g}\left|\nu_{l}\right| B\right)}-\exp \left(-s W_{k}^{\prime} \bar{g}\left|\nu_{l}\right| B\right)+\exp \left(+s W_{k}^{\prime} \bar{g}\left|\nu_{l}\right| B\right)\right]
\end{aligned}
$$

where the last exponential is due to the unstable mode. For $Z_{k} k^{2}<W_{k}^{\prime} \bar{g}\left|\nu_{l}\right| B$ it makes the $s$-integration divergent at the upper (i.e. IR) limit. In conventional calculations of the one-loop effective action this creates a problem from the outset, because one attempts to work at $k^{2} \rightarrow 0$ there. In the present formulation everything is well defined for $k^{2}$ sufficiently large, and one interesting question is how the renormalization group flow behaves as one approaches $k^{2} \approx \theta$ from above.

In the UV limit $s \rightarrow 0$ the terms inside the square bracket in (E.3) behave as

$$
[\ldots]=\frac{d}{2 \bar{g}\left|\nu_{l}\right| B W_{k}^{\prime}\left(B^{2} / 2\right)} \cdot \frac{1}{s}+O(s)
$$

While the $O(s)$-terms do not lead to UV-divergences for $d<6$, the term $\sim 1 / s$ leads to a divergent contribution to the proper-time integral. Though the factor $\bar{g}\left|\nu_{l}\right| B$ cancels against a similar one coming from the density of states, this divergent piece is still field-dependent because of the $B$-dependence of $W_{k}^{\prime}$. This UV divergence shows a failure of the truncation for the mass-type cutoff function $R_{k}=Z_{k} k^{2}$. The latter may be used only together with the approximation $W_{k}^{\prime \prime}=0$ on the r.h.s. of the flow equation. The divergent piece in the proper-time integral is an irrelevant constant then.

Using (E.3) and a similar formula for the scalar traces in eq. (2.5), we obtain (up to an irrelevant constant and for $\tilde{Z}_{k}=1 / \alpha_{k}, \tilde{\eta}=0$ )

$$
\begin{aligned}
& \frac{\partial}{\partial t} W_{k}\left(\frac{1}{2} B^{2}\right)=(4 \pi)^{-d / 2} \sum_{l=1}^{N^{2}-1} \bar{g}\left|\nu_{l}\right| B k^{2} \int_{0}^{\infty} d s s^{1-d / 2} \\
& \cdot\left\{(2-\eta) Z_{k}\left(W_{k}^{\prime}\right)^{1-\frac{d}{2}}\left[\frac{d-1}{2 \sinh \left(s W_{k}^{\prime} \bar{g}\left|\nu_{l}\right| B\right)}+2 \sinh \left(s W_{k}^{\prime} \bar{g}\left|\nu_{l}\right| B\right)\right] e^{-s Z_{k} k^{2}}\right. \\
& \left.-\frac{\exp \left(-s k^{2}\right)}{\sinh \left(s \bar{g}\left|\nu_{l}\right| B\right)}\right\}
\end{aligned}
$$


This evolution equation is the analogue of (2.13) with the additional assumption $W_{k}^{\prime \prime}=0$. Contrary to the Euler-McLaurin series it is valid even for strong fields $\bar{g} B \approx k^{2}$.

For $\bar{g} B \ll k^{2}$ the r.h.s. of $(\mathbb{E} .5)$ can be expanded in powers of $B$. Apart from the different form of $R_{k}$, this reproduces the Euler-McLaurin expansion. Expanding up to order $B^{4}$ we find, for instance,

$$
\frac{\partial}{\partial t}\left(\frac{w_{2}}{g^{2}}\right)=4 \frac{w_{2}}{g^{2}}+\frac{127}{360 \pi^{2}} \tau_{2} r_{0}^{4,2}
$$

with $r_{0}^{4,2}=2$. This result is the counterpart of eq. (3.10) which had been obtained with the exponential cutoff for which $r_{0}^{4,2}=1 / 6$. In accordance with (2.15) we find an additional factor of 12 in the second term on the r.h.s. of (E.6). Hence also the value of the fixed point $w_{2 *}(k)$ is 12 times larger than the result (3.11). In view of the discussion following (3.13) this means that for the mass-type cutoff $R_{k}=Z_{k} k^{2}$ this higher-order correction is much larger than for the exponentially decreasing $R_{k}$ of (1.2). This is probably closely related to the ultraviolet problems and indicates that, though computationally more difficult to handle, the exponential cutoff (1.2) should be used for reliable estimates.

\section{Appendix $\mathrm{F}$}

In this appendix we derive the flow equation in the $F^{6}$ truncation. We start from the evolution equation for $\ddot{w}(\vartheta)$ which follows from differentiating (3.5) with respect to $\vartheta$ :

$$
\begin{aligned}
& \frac{\partial}{\partial t} \ddot{w}=(4+\eta) \ddot{w}+4 \vartheta w^{(3)} \\
& -(2-\eta) v_{d} g^{2} \dot{w}^{-\frac{d}{2}}\left\{(d-2) \sum_{m=1}^{\infty} \tau_{m}\left(C_{m}^{d}-E_{m}\right) r_{0}^{d, m}\right. \\
& {\left[\left(2 \vartheta \dot{w}^{2}\right)^{m-1}\left(((8-d) m-d) \dot{w} \ddot{w}+\left((4-2 d) m-d+\frac{d^{2}}{2}\right) \vartheta \ddot{w}^{2}+(4 m-d) \vartheta \dot{w} w^{(3)}\right)\right.}
\end{aligned}
$$




$$
\begin{aligned}
& \left.+2(m-1)\left(2 \vartheta \dot{w}^{2}\right)^{m-2} \dot{w}^{2}(\dot{w}+2 \vartheta \ddot{w})(2 m \dot{w}+(4 m-d) \vartheta \ddot{w})\right] \\
& +r_{1}^{d}\left[\frac{4 w^{(3)}+2 \vartheta w^{(4)}}{\dot{w}+2 \vartheta \ddot{w}}-\frac{4\left(\ddot{w}+\vartheta w^{(3)}\right)\left(3 \ddot{w}+2 \vartheta w^{(3)}\right)+2 \vartheta \ddot{w}\left(5 w^{(3)}+2 \vartheta w^{(4)}\right)}{(\dot{w}+2 \vartheta \ddot{w})^{2}}\right. \\
& +\frac{4 \vartheta \ddot{w}\left(3 \ddot{w}+2 \vartheta w^{(3)}\right)^{2}}{(\dot{w}+2 \vartheta \ddot{w})^{3}} \\
& \left.-d \frac{2 \ddot{w}^{2}+3 \vartheta \ddot{w} w^{(3)}}{\dot{w}(\dot{w}+2 \vartheta \ddot{w})}+\frac{3}{2} d \frac{\vartheta \ddot{w}^{3}}{\dot{w}^{2}(\dot{w}+2 \vartheta \ddot{w})}+2 d \frac{\vartheta \ddot{w}^{2}\left(3 \ddot{w}+2 \vartheta w^{(3)}\right)}{\dot{w}(\dot{w}+2 \vartheta \ddot{w})^{2}}\right] \\
& \left.+\frac{1}{4} d(d-1)(d-2)\left(N^{2}-1\right) r_{2}^{d}\left[\frac{w^{(3)}}{\dot{w}}-\frac{d+2}{2} \frac{\ddot{w}^{2}}{\dot{w}^{2}}\right]\right\} \\
& +8(d-2) v_{d} g^{2} \sum_{m=1}^{\infty} m(m-1) \tau_{m} E_{m} r_{0}^{d, m}(2 \vartheta)^{m-2}
\end{aligned}
$$

Evaluating this equation for $\theta=0$ yields eq. (3.18). Taking one further $\vartheta$-derivative at $\vartheta=0$ we obtain for $d=4$ and $k>k_{n p}$ the flow equation for $w_{3}$ (with $C_{2}^{4}-E_{2}=$ $-\frac{29}{80}, C_{3}^{4}-E_{3}=-\frac{137}{10080}$ and $\left.E_{3}=\frac{31}{30240}\right)$ :

$$
\begin{aligned}
& \frac{\partial}{\partial t} w_{3}=(8+\eta) w_{3} \\
& +\frac{g^{2}}{16 \pi^{2}}\left\{\left(\frac{442}{315}-\frac{137}{210} \eta\right) \tau_{3} r_{0}^{4,3}+\frac{87}{5}(2-\eta) \tau_{2} r_{0}^{4,2} w_{2}\right. \\
& -(2-\eta) r_{1}^{4}\left(3 w_{4}-51 w_{2} w_{3}+105 w_{2}^{3}\right) \\
& \left.-3(2-\eta)\left(N^{2}-1\right) r_{2}^{4}\left(w_{4}-9 w_{2} w_{3}+12 w_{2}^{3}\right)\right\}
\end{aligned}
$$

For the infrared cutoff (1.2) one has $r_{0}^{4,2}=\frac{1}{6}, r_{0}^{4,3}=-\frac{1}{30}$ and we observe that the perturbatively leading term $\sim g^{2}$ is negative. This implies a positive perturbative fixpoint value for the ratio $w_{3} / g^{2}$.

This discussion can easily be generalized for arbitrary $w_{n}$. In lowest order in $g^{2}$ the flow equation (F.1) simplifies considerably $\left(k>k_{n p}\right)$

$$
\begin{aligned}
& \frac{\partial}{\partial t} \ddot{w}=4 \ddot{w}+4 \vartheta w^{(3)} \\
& -8(d-2) v_{d} g^{2} \sum_{m=2}^{\infty} m(m-1) \tau_{m} r_{0}^{d, m}\left(C_{m}^{d}-2 E_{m}\right)(2 \vartheta)^{m-2}
\end{aligned}
$$

This implies for $n \geq 2$ the flow equations

$$
\frac{\partial}{\partial t} w_{n}=4(n-1) w_{n}-(d-2) v_{d} g^{2} 2^{n+1} n ! \tau_{n} r_{0}^{d, n}\left(C_{n}^{d}-2 E_{n}\right)
$$


and the infrared fixed point values

$$
\left(\frac{w_{n}}{(d-2) v_{d} g^{2}}\right)_{*}=\frac{2^{n-1}}{n-1} n ! \tau_{n} r_{0}^{d, n}\left(C_{n}^{d}-2 E_{n}\right)
$$

For $d=4$ and with (2.16) this yields

$$
\begin{aligned}
w_{n *} & =d_{n} \frac{g^{2}}{16 \pi^{2}} \\
d_{n} & =\frac{2^{n} n !}{(n-1)(2 n-1) !} \tau_{n} B_{2 n-2}\left(\frac{2^{2 n-1}-1}{2 n} B_{2 n}-1\right)
\end{aligned}
$$

where for $S U(2)$

$$
\frac{d_{2}}{\tau_{2}}=-\frac{127}{540}, \quad \frac{d_{3}}{\tau_{3}}=\frac{221}{37800}
$$

The series of $d_{n}$ is alternating as long as the bracket is dominated by -1 .

The infrared fixed point in $w_{2} / g^{2}$ implies that the coefficient $W_{2}$ in (3.1) diverges $\sim k^{-4}$

$$
W_{2} \sim-\frac{\bar{g}^{4}}{k^{4}}
$$

and similar for $W_{3}$. Because of the infrared divergence for $k \rightarrow 0$, a result of this type could never have been found in standard perturbation theory. It could, however, be derived using the " $\frac{D}{D t} \approx \frac{\partial}{\partial t}$ "-approximation of the effective average action which we displayed in eq. (A.29). For this purpose one can neglect the $W_{k}^{\prime \prime}$ terms on the r.h.s. of (A.29) and approximate $W_{k}^{\prime}=w_{1}$. Then, with (B.2) inserted into (A.29), one obtains for the $k$-dependent terms in $\Gamma_{k}$ :

$$
\begin{aligned}
\Gamma_{k}[A, A]= & \frac{1}{2} \operatorname{Tr}_{x c L} \ln \left[w_{1} \mathcal{D}_{T}+\left(w_{1}-\frac{1}{\alpha_{k}}\right) D \otimes D+R_{k}\left(w_{1} \mathcal{D}_{T}+\left(w_{1}-\frac{1}{\alpha_{k}}\right) D \otimes D\right)\right] \\
& -\operatorname{Tr}_{x c} \ln \left[-D^{2}+R_{k}\left(-D^{2}\right)\right]
\end{aligned}
$$

If one extracts the $F_{\mu \nu}^{4}$-term from these traces, one finds a (renormalized) coefficient which equals exactly $W_{2}$, as extracted from the fixed point (3.11). Clearly (3.8) goes beyond the " $\frac{D}{D t} \approx \frac{\partial}{\partial t}$ " approximation. The terms proportional to $\eta$ and to $w_{2}^{2}$ could not have been obtained in this approximation. 
Finally, for $k<k_{n p}$ the flow equation for $w_{3}$ follows from (F.1) as

$$
\begin{aligned}
& \frac{\partial}{\partial t} w_{3}=8 w_{3}+\frac{g^{2}}{16 \pi^{2} w_{1}^{2}}\left\{\tau_{3} r_{0}^{4,3}\left(\frac{137}{105} w_{1}^{6}+\frac{31}{315} w_{1}^{2}\right)+\frac{174}{5} \tau_{2} r_{0}^{4,2} w_{1}^{3} w_{2}\right. \\
& -6 r_{1}^{4}\left(\frac{w_{4}}{w_{1}}-17 \frac{w_{2} w_{3}}{w_{1}^{2}}+35 \frac{w_{2}^{3}}{w_{1}^{3}}\right) \\
& \left.-6\left(N^{2}-1\right) r_{2}^{4}\left(\frac{w_{4}}{w_{1}}-9 \frac{w_{2} w_{3}}{w_{1}^{2}}+12 \frac{w_{2}^{3}}{w_{1}^{3}}\right)\right\}
\end{aligned}
$$




\section{References}

[1] G. K. Savvidy, Phys. Lett. 71B (1977) 133

[2] W. Dittrich and M. Reuter, Phys. Lett. B128 (1983) 321;

M. Reuter and W. Dittrich, Phys. Lett. B144 (1984) 99;

H. D. Trottier, Phys. Rev. D44 (1991) 464

[3] C. Wetterich, Nucl. Phys. B352 (1991) 529;

C. Wetterich, Z. Phys. C57 (1993) 451; C60 (1993) 461

[4] C. Wetterich, Phys. Lett. B301 (1993) 90

[5] F. Wegner, A. Houghton, Phys. Rev. A8 (1973) 401;

K. G. Wilson, I. G. Kogut, Phys. Rep. 12 (1974) 75;

S. Weinberg, Critical Phenomena for Field Theorists, Erice Subnucl. Phys. (1976) 1 ;

J. Polchinski, Nucl. Phys. B231 (1984) 269;

A. Hasenfratz, P. Hasenfratz, Nucl. Phys. 270 (1986) 685

[6] M. Bonini, M. D'Attanasio, and G. Marchesini, Nucl. Phys. B409 (1993) 441;

C. Wetterich, Int. J. Mod. Phys. A9 (1994) 3571;

U. Ellwanger, Z. Phys. C62 (1994) 503;

T. R. Morris, Phys. Lett. B329 (1994) 241

[7] M. Reuter and C. Wetterich, Nucl. Phys. B417 (1994) 181

[8] M. Reuter in: Proceedings of the 5th Hellenic School and Workshops on Elementary Particle Physics, Corfu, Greece, 1995; hep-th/9602012

[9] M. Reuter, DESY 96/065 and hep-th/9604124 
[10] C. Becchi, On the construction of renormalized quantum field theory using renormalization group techniques, in: Elementary Particles, Field Theory and Statistical Mechanics, eds. M. Bonini, G. Marchesini, and E. Onofri, Parma University, 1993;

M. Bonini, M. D'Attanasio, and G. Marchesini, Nucl. Phys. B418 (1994) 81; B421 (1994) 429; B437 (1995) 163, Phys. Lett. B346 (1995) 87

[11] U. Ellwanger, Phys. Lett. B335 (1994) 364;

U. Ellwanger, M. Hirsch, and A. Weber, preprint LPTHE Orsay 95-39

[12] M. Reuter and C. Wetterich, Phys. Lett. B334 (1994) 412

[13] U. Ellwanger and C. Wetterich, Nucl. Phys. B423 (1994) 137

[14] M. Reuter and C. Wetterich, Nucl. Phys. B391 (1993) 147; Nucl. Phys. B408 (1993) 91

[15] M. Reuter and C. Wetterich, Nucl. Phys. B427 (1994) 291

[16] L. F. Abbott, Nucl. Phys. B185 (1981) 189;

W. Dittrich and M. Reuter, Selected Topics in Gauge Theories, Lecture Notes in Physics, Vol. 244, Springer, Berlin, 1986

[17] S. L. Adler, Phys. Rev. D23 (1981) 2905; Nucl. Phys. B217 (1983) 381;

S. L. Adler and T. Piran, Phys. Lett. 113B (1982) 405; 117B (1982) 91;

H. Hata, Y. Kazama, Nucl. Phys. B232 (1984) 85

[18] N. K. Nielsen and P. Olesen, Nucl. Phys. B144 (1978) 376; Phys. Lett. 79B (1978) 304

[19] H. J. Kaiser, K. Scharnhorst, and E. Wieczorek, J. Phys. G16 (1990) 161 
[20] W. Dittrich and M. Reuter, Effective Lagrangians in Quantum Electrodynamics, Lecture Notes in Physics, Vol. 220, Springer, Berlin, 1985

[21] J. A. Zuk, J. Phys. A: Math. Gen. 18 (1985) 1795;

C. M. Fraser, Z. Phys. C28 (1985) 101;

R. I. Nepomechie, Phys. Rev. D31 (1985) 3291

[22] M. G. Schmidt and C. Schubert, Phys. Lett. B318 (1993) 438, Phys. Lett. B331 (1994) 69;

D. Fliegner, M. G. Schmidt, and C. Schubert, Z. Phys. C64 (1994) 111

[23] H. G. Dosch, Lectures at Varenna School '95, Course XIII, p. 279, eds. A. DiGiacomo and D. Diakonov (1996);

A. DiGiacomo and H. Panagopoulos, Phys. Lett. B285 (1992) 133;

M. D'Elia, A. DiGiacomo, and E. Meggiolaro, hep-lat/9705032

[24] L. O'Raifeartaigh, A. Wipf, and H. Yoneyama, Nucl. Phys. B271 (1986) 653;

A. Ringwald and C. Wetterich, Nucl. Phys. B334 (1990) 506;

N. Tetradis and C. Wetterich, Nucl. Phys. B383 (1992) 197

[25] L. O'Raifeartaigh, Group structure of gauge theories, Cambridge University Press, Cambridge, 1986 